\title{
Study of the Largest Multiwavelength Campaign of the Microquasar GRS 1915+105
}

\author{
Y. Ueda ${ }^{1}$, K. Yamaoka ${ }^{2}$, C. Sánchez-Fernández ${ }^{3}$, V. Dhawan ${ }^{4}$, S. Chaty $^{5}$, \\ J.E. Grove ${ }^{6}$, M. McCollough ${ }^{7}$, A.J. Castro-Tirado ${ }^{3,8}$, F. Mirabel ${ }^{9,10}$, \\ K. Kohno ${ }^{11,12}$, M. Feroci ${ }^{13}$, P. Casella ${ }^{13}$, S.A. Trushkin ${ }^{14}$, H. Castaneda ${ }^{15}$, \\ J. Rodríguez ${ }^{9}$, P. Durouchoux ${ }^{9}$, K. Ebisawa ${ }^{16}$, T. Kotani ${ }^{16}$, J. Swank ${ }^{16}$, H. Inoue $^{1}$
}

\begin{abstract}
\footnotetext{
${ }^{1}$ Institute of Space and Astronautical Science, Sagamihara 229-8510, Japan

${ }^{2}$ Institute of Physical and Chemical Research (RIKEN), Wako 351-01, Japan

${ }^{3}$ LAEFF-INTA, Madrid, Spain

${ }^{4} \mathrm{NRAO}, \mathrm{USA}$

${ }^{5}$ Department of Physics and Astronomy, The Open University, UK

${ }^{6}$ Naval Research Lab, USA

${ }^{7} \mathrm{MSFC} / \mathrm{NASA}$, USA

${ }^{8}$ IAA-CSIC, Granada, Spain

${ }^{9}$ Service d'Astrophysique, Saclay, France

${ }^{10}$ IAFE/CONICET, Argentina

${ }^{11}$ Nobeyama Radio Observatory, NAO, Japan

${ }^{12}$ Institute of Astronomy, The University of Tokyo, Japan

${ }^{13}$ Istituto di Astrofisica Spaziale, C.N.R., Italy

${ }^{14} \mathrm{SAO}$, Russia

${ }^{15}$ Observatorio Astronomico Nacional de Mexico, Mexico

${ }^{16}$ GSFC/NASA, USA
}

We present the results from a multiwavelength campaign of GRS 1915+105 performed from 2000 April 16 to 25. This is one of the largest coordinated set of observations ever performed for this source, covering the wide energy band in radio $(13.3-0.3 \mathrm{~cm})$, near-infrared $(J-H-K)$, $\mathrm{X}$-rays and Gamma-rays (from $1 \mathrm{keV}$ to $10 \mathrm{MeV}$ ). During the campaign GRS 1915+105 was predominantly in the "plateau" (or low/hard) state but sometimes showed soft X-ray oscillations: before April 20.3, rapid, quasi-periodic $(\simeq 45 \mathrm{~min})$ flare-dip cycles were observed. In the 
spectral energy distribution in the plateau state, optically-thick synchrotron emission and Comptonization is dominant in the radio and $\mathrm{X}$ to Gamma-ray bands, respectively. The small luminosity in the radio band relative to that in X-rays indicates that GRS 1915+105 falls in the regime of "radio-quiet" microquasars. In three epochs we detected faint flares in the radio or infrared bands with amplitudes of 10-20 mJy. The radio flares observed on April 17 shows frequency-dependent peak delay, consistent with an expansion of synchrotron-emitting region starting at the transition from the hard-dip to the soft-flare states in X-rays. On the other hand, infrared flares on April 20 appear to follow (or precede) the beginning of X-ray oscillations with an inconstant time delay of $\simeq 5-30$ min. This implies that the infrared emitting region is located far from the black hole by $\gtrsim 10^{13} \mathrm{~cm}$, while its size is $\lesssim 10^{12} \mathrm{~cm}$ constrained from the time variability. We find a good correlation between the quasisteady flux level in the near-infrared band and in the X-ray band. From this we estimate that the reprocessing of X-rays, probably occurring in the outer parts of the accretion disk, accounts for about $20-30 \%$ of the observed $K$ magnitude in the plateau state. The OSSE spectrum in the 0.05-10 MeV band is represented by a single power law with a photon index of 3.1 extending to $\sim 1 \mathrm{MeV}$ with no cutoff. We can model the combined GIS-PCA-HEXTE spectra covering 1-200 keV by a sum of the multi-color disk model, a broken power law modified with a high energy cutoff, and a reflection component. The power-law slope above $\sim 30 \mathrm{keV}$ is found to be very similar between different states in spite of large flux variations in soft X-rays, implying that the electron energy distribution is not affected by the change of the state in the accretion disk.

Subject headings: accretion, accretion disks — black hole physics - infrared: stars - radio continuum: stars — stars: individual (GRS 1915+105) - X-rays: stars 


\section{Introduction}

Up to present a few superluminal jet sources have been identified in our Galaxy (for a review see Mirabel \& Rodríguez 1999): GRS 1915+105, GRO J1655-40, possibly XTE J1748288, XTE J1550-564 (Hannikainen et al. 2001), and V4641 Sgr (Orosz et al. 2001). The similarity of the observed properties in these sources to those of AGNs indicates that the underlying physics is common to a wide mass range of the central black hole from $\sim 10 \mathrm{M}_{\odot}$ to $\sim 10^{9} \mathrm{M}_{\odot}$, except for scaling in luminosity, size, and variability, which are proportional to the black hole mass. These objects, so-called "microquasars", are ideal laboratories for understanding the mechanism of relativistic jets and its relation with accretion flow onto a black hole, owing to the brightness in X-ray bands and fast variability. Studies in multiwavelength bands are particularly important, because with X-rays and Gamma-rays we can probe into the innermost part of the accretion disk, while in radio and infrared bands we can trace ejection of relativistic jets.

GRS 1915+105 was discovered in 1992 as an X-ray transient by WATCH / GRANAT (Castro-Tirado, Brandt,\& Lund 1992; CastroTirado et al. 1994). Later, Mirabel \& Rodríguez (1994) detected from this source the first superluminal motion in our Galaxy. By assuming a distance of $12.5 \pm 1.5 \mathrm{kpc}$ estimated from H I absorption, the kinematics of the two-sided jets revealed its intrinsic velocity of $(0.92 \pm 0.08) c$ and the inclination of $70 \pm 2^{\circ}$ (Mirabel \& Rodríguez 1994). The above distance has been confirmed by Chaty et al. (1996) with millimeter observations and revised by Dhawan, Gross, \& Rodríguez (2000) to be $12 \pm 1 \mathrm{kpc}$ from an updated H I spectrum, although the uncertainty may be larger than $1.5 \mathrm{kpc}$ (see Chaty et al. 2001). Similar jet parameters $\left(0.98 c\right.$ and $66^{\circ}$ at $\left.11 \mathrm{kpc}\right)$ were obtained from other ejection events in 1997 October / November (Fender et al. 1999). Recently, Greiner et al. (2001) have identified the companion to be a K-M III star from the infrared spectroscopic observations, classifying GRS $1915+105$ as a low mass X-ray binary, as earlier proposed by Castro-Tirado, Geballe \& Lund (1996). The mass of the black hole has been determined to be $14 \pm 4 \mathrm{M}_{\odot}$ (Greiner, Cuby, \& McCaughrean 2001). In X-rays, GRS 1915+105 shows dramatic temporal/spectral variations occurring in regular cycles (e.g., Belloni et al. 2000). Simultaneous observations in radio, infrared, and X-ray bands indicated a close connection between accretion disk instabilities and ejection of plasmoids emitting via synchrotron radiation (e.g., Pooley \& Fender 1997; Mirabel et al. 1998; Eikenberry et al. 1998).

In this paper, we report results of a multiwavelength campaign of GRS 1915+105 performed from 2000 April 16 to April 25. This is one of the largest coordinated set of observations ever performed for this source, covering the wide energy band in radio $(\lambda=13.3-0.3$ $\mathrm{cm})$, infrared $(J, H, K)$, X-rays and Gammarays (from $1 \mathrm{keV}$ to $10 \mathrm{MeV}$ ), during a period of 9 days in total. Results of the Chandra HETGS observation made on 2000 April 24 are reported by Lee et al. (2002), which are not included here. We report a summary of the coordinated observations in $\S 2$. Then, data analysis and highlights of the results obtained from this campaign are presented in $\S 3$, followed by discussion on three topics in $\S 4$. In $\S 5$, we summarize the conclusion.

\section{Observations}

We conducted the multiwavelength campaign when the $A S C A$ satellite performed a 
long look at GRS 1915+105 from April 17.5 to April 25.5 (UT throughout the paper). Simultaneous observations were made with Rossi $X$-ray Timing Explorer (RXTE) on April 17, 20, 22, 23, 24, and 25, with BeppoSAX from April 21 to 24, and with OSSE / the Compton Gamma Ray Observatory (CGRO) from April 18 to 26 . From ground facilities, radio observations were made at the Very Large Array (VLA) 凹 (observers: Mirabel and Dhawan) on April 17, 22, and 23, and at the Nobeyama Millimeter Array (NMA) from April 17 to 19 (Kohno), and infrared observations at the Calar Alto Observatory from April 18 to 25 (Castro-Tirado and Sánchez-Fernández) and at the European Southern Observatory (ESO) from April 17 to 25 (Chaty). In addition, monitoring data of the Green Bank Interferometer (=GBI, $2.25 \mathrm{GHz}$ and $8.3 \mathrm{GHz}$ ), All Sky Monitor (ASM) / RXTE (1.5-12 $\mathrm{keV})$, and BATSE / CGRO (20-100 keV) were available. Rodríguez et al. (2001) report timing analysis from $R X T E$ observations on April 17, 22, and 23, and Feroci et al. (2001) report the BeppoSAX results. More detailed analysis of the infrared $K^{\prime}$ data taken at Calar Alto is reported in Sánchez-Fernández (2001). Below, we summarize observations and data reduction for each observation. Table 1 gives a log of all the observations.

\subsection{CGRO Observations}

\subsubsection{OSSE}

The OSSE instrument on the CGRO observed GRS 1915+105 over the period April

${ }^{17}$ The NRAO VLA is a facility of the National Science Foundation, operated under Cooperative Agreement by Associated Universities, Inc.

${ }^{18}$ Based on observations collected at the European Southern Observatory, Chile (ESO No 65.H-0247).
2000 18.7-25.6. OSSE covered the low-energy gamma-ray band, from $50 \mathrm{keV}$ to $10 \mathrm{MeV}$ with good spectral resolution. It had a $3.8^{\circ} \times$ $11.4^{\circ}$ field of view (at $50 \mathrm{keV}$ ). Energy spectra were accumulated in an alternating sequence of 2-minute measurements of source and background fields (Johnson et al. 1993). For these observations, the long axis of the collimator was inclined with respect to the galactic plane, and the background fields were chosen to minimize and compensate for any contamination from Galactic diffuse emission.

The observations discussed here were performed late in the $C G R O$ mission, following the last altitude reboost. At this high altitude, the OSSE instrument was subject to additional activation in the South Atlantic Anomaly (SAA). The radioactive decay of ${ }^{128} \mathrm{I}$ in the scintillator is the dominant source of internal background in the $\sim 1-2 \mathrm{MeV}$ band, which results in a flattening of the sensitivity curve and - if the background subtraction is inadequate - can create positive or negative spectral features in this band.

\subsubsection{BATSE}

The BATSE experiment onboard CGRO (Fishman et al. 1989) was used to monitor the hard X-ray emission from GRS 1915+105. The BATSE Large Area Detectors (LADs) can monitor the whole sky almost continuously in the energy range of $20 \mathrm{keV}-2 \mathrm{MeV}$ with a typical daily 3 sigma sensitivity of better than 100 mCrab. Detector counting rates with a timing resolution of 2.048 seconds are used for our data analysis. To produce the GRS 1915+105 light curve, single step occultation data were taken using a standard Earth occultation analysis technique used for monitoring hard X-ray sources (Harmon et al. 1992). Interference from known bright 
sources were removed. A spectral analysis of the BATSE data indicated that the data were well fit by a power law with a spectral index of -2.8 . The single occultation step data were then fit with a power law with this index to determine daily flux measurements in the 20 $100 \mathrm{keV}$ band.

\subsection{RXTE Observations}

During the campaign, 8 pointing observations were made by $R X T E$ on 2000 April $17,20,22,23,24$, and 25 , covering the 2 $60 \mathrm{keV}$ band with the Proportional Counter Array (PCA: Jahoda et al. 1996) and 15$250 \mathrm{keV}$ band with the High Energy X-ray Timing Experiment (HEXTE: Rothschild et al. 1998). For data reduction and analysis, we used the FTOOLS V5.0 package provided by GSFC/NASA. The good time intervals (GTIs) of the PCA data were selected on the two criteria: (1) the elevation angle was $10^{\circ}$ or larger, and (2) the offset pointing was less than $0.02^{\circ}$. For spectral analysis, we used the standard2 mode data, which has 129 energy channels with a time resolution of $16 \mathrm{~s}$. We used only events of the top layer from PCU0, PCU2, and PCU3, which were always on during the campaign except for April 25.24-25.26, when PCU3 was temporarily turned off. The background spectrum was constructed from the standard model prepared for bright sources, which includes contribution of both the cosmic X-ray background and particle background. The HEXTE data were selected with the same GTIs as the PCA. We used the standard mode data and subtracted the background taken from the rocking motion. Data of cluster-0 and cluster- 1 are summed. Besides the pointing data with the PCA and HEXTE, we also used the ASM data in the $1.5-12 \mathrm{keV}$ band 19 .

\subsection{BeppoSAX Observations}

BeppoSAX joined the campaign as a part of the on-going program for Target of Opportunity observations on this source. The Narrow Field Instruments (NFI, 0.1-300 keV, Boella et al. 1997a) observed the source from April 21 19:09 (MJD 51655.798) to April 24 11:32 (MJD 51658.480) for a net exposure of $80 \mathrm{ks}$ in the Medium Energy Concentrator Spectrometer (MECS, 2-10 keV, Boella et al. 1997b) and $76 \mathrm{ks}$ in the Phoswich Detection System (PDS, 15-300 keV, Frontera et al. 1997). The two large data gaps are due to the temporary suspension of the BeppoSAX operation during the nights of the week-ends occurred in 2000. Unfortunately, this limitation to the satellite operations prevented the simultaneous observations with some groundbased telescopes. Standard data reduction and cleaning procedures (Fiore et al. 1999) were applied in order to extract the final scientific products. The source appears bright during the entire observation, with an average count rate of about 100 counts $\mathrm{s}^{-1}$ in the MECS instrument $(2-10 \mathrm{keV})$ and 30 counts $\mathrm{s}^{-1}$ in the PDS $(15-300 \mathrm{keV})$.

\section{4. $A S C A$ Observations}

We observed GRS $1915+105$ with the $A S C A$ satellite (Tanaka, Inoue \& Holt 1994) in the 0.7-10 keV band from April 17.5 to 25.5. A net exposure of $271 \mathrm{ksec}$ is achieved, the longest continuous observation performed with $A S C A$ for this source. ASCA carries four $\mathrm{X}$-ray telescopes, coupled with the two Gas Imaging Spectrometers (Ohashi et al. 1996)

\footnotetext{
${ }^{19}$ provided by the ASM team at MIT and NASA GSFC
} $\mathrm{SOF} / \mathrm{GOF}$ through the web 
and two Solid-state Imaging Spectrometers (Yamashita et al. 1999). Throughout the observations, the GIS was operated in the standard pulse height mode with the nominal bit assignment, and the SIS was operated in the 1-CCD Bright mode.

In this paper, we present only results of the GIS data, since extremely careful treatment is required for the SIS data, which suffered from severe pile-up as well as degradation of the response due to integrated radiation damage at the end of the $A S C A$ missions. We only use data taken in the high bit-rate telemetry mode, except when examining the presence of flare events from light curves (see below). In the medium bit-rate, dead time becomes significant and monitor counts measured by nonreset counter (such as L1) sometimes spilled over due to high count rate from the source, which makes it difficult to apply dead-time correction. The GIS data were selected with the criteria of (1) the elevation angle of the source from the Earth limb was higher than 5 degrees and (2) the satellite was not in the SAA. This caused data gaps with a period of 90 minutes due to the satellite orbit. We calculate the GIS spectra using events in a circular region within a radius of $6^{\prime}$ centered at the peak. The energy scale of each detector were finely tuned using the instrumental structure of the Gold M-edge and Xenon Ledge. We estimate the resultant accuracy of the absolute gain to be about $0.5 \%$.

\subsection{Near-Infrared Observations at the ESO}

Near-infrared observations of GRS 1915+105 were performed with the $3.58 \mathrm{~m}$ New Technology Telescope (NTT) at the European Southern Observatory (ESO) at La Silla, Chile, during April 19-25, 2000. The telescope was equipped with the infrared spectrograph and imaging camera Son of ISAAC (SOFI). The observations were performed in the course of an on-going program of Target of Opportunity observations on Galactic hard X-ray sources (PI: Chaty).

\subsubsection{Imaging}

In imaging the broad band filters $J, H$, and $K s$ were used, in combination with the Large Field, giving a $4.9 \times 4.9 \mathrm{arcmin}^{2}$ field of view, with a plate scale of 0.292 arc-seconds pixel $^{-1}$. The J-band filter is centered on 1.247 $\mu \mathrm{m}$, with a width of $0.290 \mu \mathrm{m}$, representing $23 \%$ of the wavelength, the $H$-band filter is centered on $1.653 \mu \mathrm{m}$, with a width of 0.297 $\mu \mathrm{m}$, representing $18 \%$ of the wavelength, and finally the $K s$-band filter is centered on 2.162 $\mu \mathrm{m}$, with a width of $0.275 \mu \mathrm{m}$, representing $13 \%$ of the wavelength.

On each night of the April 19th, 20th, 21st and 25 th, the $J, H$, and $K s$ band images were acquired with an integration time of 60s: four frames of 15 s each were averaged for the $J$ and $H$, and six frames of 10 s each for the $K s$. The combined magnitudes given for these nights in Table 2 are the result of 9 co-added and median filtered 60s exposures, with random offsets and direction between each exposure. The conditions were photometric for most of the campaign, the seeing being typically 0.8 arcsec.

Concerning the nights of the 23rd and 24th, we continuously observed in the $K s$ band, taking 60 exposures with an integration time of 60 s each, by averaging $6 \times 10$ s frames, and randomly offseting in distance and direction between each frame, during nearly 1.5 hours. The combined magnitudes given for these nights in Table 2 are the result of the 60 co-added and median filtered 60s exposures. 
A total of 229 60s-frames were acquired during those 6 nights.

The images were processed using IRAF reduction software. Each of the images were corrected by a normalized dome-flat field, and sky-subtracted by a sky image created from combining with a median filter a total of 9 consecutive images. The data were then analyzed using the IRAF reduction task "apphot", taking different apertures depending on the photometric conditions of the night.

Absolute photometry was performed using 2 standard stars from the new system of faint near-infrared standard stars (Persson et al. 1998): No. 9164 (HST P565-C) and 9178 (HST S808-C). Each exposure of these standard stars is the average of $7 \times 1.2 \mathrm{~s}$ integration time frames, and this is repeated 5 times by offsetting the images of 1 arcmin to the North-West, North-East, South-East and South-West from the central position, and the final image is the co-add and median filter of those individual frames. The Zero-points in $J, H$, and $K s$ during 19-25th of April were respectively $1.877 \pm 0.008,2.096 \pm 0.040$ and $2.652 \pm 0.062$ magnitudes.

\subsubsection{Spectroscopy}

On April 22, we also performed some low resolution $(R=600)$ 1.53-2.52 $\mu \mathrm{m}$ wavelength range spectroscopy with a fixed width slit of 1 arcsecond, using the GR Grism Red at 1st order, giving a dispersion of 10.22, and a resolution of 1000 .

We acquired 10 exposures of $2 \times 120$ s each on GRS 1915+105, giving a total exposure time of 40 minutes. We also acquired 8 exposures of $3 \times 10$ s each on the spectrophotometric standard Hip 95550, giving a total exposure time of 4 minutes. We corrected the images by a dome-flat field, and sky-subtracted the spectra by offset spectra. We wavelengthcalibrated the images thanks to a Xenon lamp spectrum acquired with the Red Grism and the same setup. We thereafter normalized the spectra.

\subsection{Near-infrared Observations at the Calar Alto Observatory}

Infrared $K^{\prime}$ observations of GRS $1915+105$ were performed with the $1.23 \mathrm{~m}$ Telescope at the German-Spanish Observatory at Calar Alto, Spain, during April 17-24, 2000. The telescope is equipped with the $\mathrm{f} / 8 \mathrm{Mpi}$ Für Astronomie General-Purpose Infrared Camera (MAGIC) which provides a 5-arcmin field of view, at a resolution of 1.2 arcsec pixel ${ }^{-1}$. The $K^{\prime}$ filter has a central wavelength of 2.10 $\mu \mathrm{m}$ with a width of $0.34 \mu \mathrm{m}$ and a quantum efficiency of about 0.6. The seeing was typically 1.5 arcsec. On each night, series of nine $45 \mathrm{~s}$ (co-added) exposures were obtained, with offsets of $\sim 15$ arcsec between each exposure. One whole night and two half-nights of the campaign were lost due to bad weather conditions. A total of 924 good frames were obtained.

The images were processed using IRAF reduction software. For each of the images, a median-combined sky image was created from a total of 9 consecutive images, time-centered in the image to be sky-subtracted. The resulting sky-subtracted images were then flatfielded with an image constructed from the difference of dome flats obtained with the flat field lamps on and off.

The data were then analyzed using the IRAF reduction task "apphot", with different apertures depending on the photometric conditions of the night. Relative photometry was performed using 5 field secondary standard stars, calibrated during one of our pre- 
vious observational campaigns on this field.

\subsection{NMA Observations}

The Nobeyama Millimeter Array observations of $3 \mathrm{~mm}$ continuum emission toward GRS 1915+105 were made on 2000 April 17, 18, and 19. The NMA consists of six $10 \mathrm{~m}$ antennas equipped with cryogenically cooled SIS tunerless receivers. The system noise temperatures toward the zenith were about $120 \mathrm{~K}$ in double side band for the first two days, yet increased to about $200 \mathrm{~K}$ in the last day due to a poor weather condition. The array was in D configuration (the most compact) for this period, and the resultant synthesized beam were about $8 " \times 6$ " at the observing frequencies i.e., $88.6 \mathrm{GHz}$ for the lower side band, and $100.6 \mathrm{GHz}$ for the upper side band. The side band separation was achieved by 90 degrees phase switching to the reference signal. The backend was the Ultra WideBand Correlator (UWBC), which is an XF type digital-spectrocorrelator with a bandwidth of $1024 \mathrm{MHz}$ (Okumura et al. 2000). The passband across the channels were calibrated by observations of $3 \mathrm{C} 454.3$, and the quasar B1923+210, which was about 3.5 Jy during the observations, was observed every 20 minutes to calibrate the temporal variation of the visibility amplitude and phase. The uncertainty in the absolute flux scale is estimated to be about $\pm 10 \%$. The raw visibility data were reduced with the UVPROC2 developed at NRO (Tsutsumi et al. 1997), and then Fourier transformed and deconvolved using a CLEAN technique implemented with the NRAO AIPS (Astronomical Image Processing System). Upper and lower side band images were averaged to enhance signal-tonoise ratios. The achieved rms noise levels after the averaging were $1.3,1.1$, and $2.1 \mathrm{mJy}$ for the three observing runs, respectively (indicated by the error bars in Figure 1).

\subsection{VLA Observations}

The VLA is a multi-frequency, multi- configuration, aperture synthesis imaging instrument, consisting of 27 antennas of $25 \mathrm{~m}$ diameter. The receivers at 5.0, 8 , and $22 \mathrm{GHz}$, were used in these observations, with 2 adjacent bands of $50 \mathrm{MHz}$ nominal bandwidth processed in continuum mode. To get simultaneous time coverage at 3 wavelengths, we used the antennas in 3 subarrays with 11, 7 and 9 antennas respectively. The corresponding 1-sigma sensitivities in 10 minutes are 0.25 , 0.15 , and $0.15 \mathrm{mJy}$ respectively for the 3 subarrays. (The rms noise is indicated by the error bars on the plots of Figure 2). The array configuration is varied every 4 months to cycle between 4 sets A, B, C, D, with maximum baselines of about 36, 11, 3.4 and $1 \mathrm{~km}$. The $\mathrm{C}$ array was in use during these observations.

For all observations reported here, GRS $1915+105$ was unresolved by the synthesized beams $(0.9 \operatorname{arcsec}$ at $\lambda=1.3 \mathrm{~cm}, 1.7$ arcsec at $3.6 \mathrm{~cm}$, and $4.8 \operatorname{arcsec}$ at $6.0 \mathrm{~cm}$ ) for any subarray and wavelength. Other sources (HII regions, see e.g., Figure 4 of Rodríguez et al. 1995 and Figure 1 of Chaty et al. 2001) are present in the field of view, mainly at $1.4 \mathrm{GHz}$. These are well separated in the images, and also are much weaker and resolved out by the narrower beam at short wavelengths, so there is no confusion with GRS 1915+105.

The primary flux density calibrators were $3 \mathrm{C} 286(1331+305)$ and $3 \mathrm{C} 48(0137+331)$ and the phase calibrator was $1925+211$. Calibration and imaging were carried out with standard tasks in the NRAO AIPS package. In practice, the flux density errors are set not by the rms receiver (thermal) noise stated 
above, but by errors in the flux density scale, estimated to be $3-5 \%$ of the measurement; and/or source variability, depending on the occasion.

\subsection{Monitoring Observations by the Green Bank Interferometer}

The radio fluxes at $2.25 \mathrm{GHz}(\lambda=3.6 \mathrm{~cm})$ and $8.3 \mathrm{GHz}(\lambda=13.3 \mathrm{~cm})$ were monitored by the Green Bank Interferometer (GBI) on a $2.4 \mathrm{~km}$ baseline with a band width of $35 \mathrm{MHz}$ for each frequency. Detailed description of the flux calibration can be found in Foster et al. (1996 and references therein). Up to 12 scans were performed every day with a 10-15 minutes integration time. The random noise $(1 \sigma)$ is estimated to be about $4 \mathrm{mJy}$ at 2.25 $\mathrm{GHz}$ and $6 \mathrm{mJy}$ at $8.3 \mathrm{GHz}$ when the flux was below $100 \mathrm{mJy}$, and the data are noisedominated below 15-20 mJy. The data on 2000 April 21 through 23 are not available due to holiday weekend shutdown. According to the operational notes rain may affect the $8.3 \mathrm{GHz}$ data on April 17.

\section{Data Analysis and Results}

\subsection{Multiwavelength Light Curves}

Multiwavelength light curves obtained from the whole observations are shown in Figure 1, sorted by wavelengths. In this figure, data with high time resolution are merged into longer time bins. For later discussions, we plot expanded light curves with higher time resolution in Figure 2 (PCA, GIS, and VLA on 2000 April 17.53-17.65), Figure 3 (PCA, GIS, and infrared $\left(K^{\prime}\right)$ on April 19.95-20.25), Figure 4 (PDS and MECS on April 23.7823.83), and Figure 5 (GIS and infrared $(H-J-$ $K s)$ on April 25.35-25.41). Below, we summarize the overall behavior of the source during the campaign.

\subsubsection{Soft $X$-rays}

It is immediately noticed from Figure 1 that before $\mathrm{UT} \simeq 2000$ April 20.3 soft X-rays $(<25 \mathrm{keV})$ showed rapid "flares" or "oscillations" in the form of quasi-periodic $(\simeq 45 \mathrm{~min}$ utes) flare-dip cycle. Detailed analysis using the 1-sec resolution data of the RXTE PCA revealed that the flare phase corresponds to Class $\alpha$ defined by Belloni et al. (2000). After April 20.3, the source entered into a more stable state, classified as Class $\chi$ (also referred as the low/hard state or the "plateau" state in the literature). Even after April 20.3, however, a few sudden, separated flares were still observed. Their epochs are marked by the arrows in the GIS light curve of Figure 1. Here, to search for as many flares as possible, we also utilized GIS LD data (one of the monitor counts; see Ohashi et al. 1996) in the bit-rate medium, which are not included in Figure 1. The presence of such flares makes the definition of the state as a function of time untrivial. 
The three flares observed after April 20.3 are essentially the same (i.e., Class $\alpha$ ) as the ones observed in the flare-dip cycles at earlier epochs. The flare that occurred at 2000 April 23.825 was clearly detected by the $B e p$ poSAX: a blow up of BeppoSAX PDS and MECS light curves is shown in Figure 4. As noticed, this flare was detected only in the MECS data $(<10 \mathrm{keV})$, with no counterpart in the high energy detector PDS. We checked for any reasonable instrumental effect that may have caused a spurious flare like the one we observed, and found none. Unfortunately, the flare occurred just before an observation gap due to Earth occultation, and therefore the study of its time history is truncated. For the portion that BeppoSAX could observe, its duration was approximately $100 \mathrm{~s}$ and the profile seems similar to the start of one $\alpha$ mode event (cf. Figure 2 and 3; Belloni et al. 2000).

The frequency of the flares changed with time, although a correct estimate is difficult because of the incomplete time coverage due to the data gaps. In particular, the source showed a periodic flare-dip cycle with a roughly constant interval of about 45 minutes between 2000 April 17.5-18.05. Then, the frequency of flares decreased and became more irregular. There is no evidence for any flare between April 19.54-19.96 at least in the available GIS or ASM data. After that the source became more active again as recognized from Figure 3. The variability pattern of the flare-dip cycle also changed with time. On April 17 (Figure 2), the duration of a dip was roughly comparable to that of oscillation between the two dips, whereas on April 20 (Figure 3 ) the duration of the dip was about 3 times longer than that of the flare. As we will show it later, the spectral and timing proper- ties in the dip phase during the flare-dip cycle observed in the earlier epochs are very similar to those in the quasi-steady, "plateau" state. Hence, we may regard that during this campaign the system was basically in the plateau state, occasionally exhibiting a single class of flares with different rates.

\subsubsection{Hard X-rays and Gamma-rays}

Throughout the observations GRS 1915+105 was quite bright in hard X-rays: the BATSE flux of $\sim 0.10$ photons $\mathrm{cm}^{-2} \mathrm{~s}^{-1}(20-100 \mathrm{keV})$ was at a typical level of the "plateau" state (Foster et al. 1996). The OSSE flux (50$300 \mathrm{keV}$ ) showed a significant variability at a $20 \%$ level (peak-to-peak) on hours to days. After April 21 when rapid soft X-ray oscillations ceased, the daily averaged flux increased by about $20 \%$. Then, it once decreased at $\mathrm{UT} \simeq 2000$ April 24.5 but recovered at April 25.0 accompanied with decrease of the soft $\mathrm{X}$-ray flux in the $0.7-10 \mathrm{keV}$ band. On much shorter time scales, hard X-ray fluxes (above $20 \mathrm{keV}$ ) is much less variable than soft $\mathrm{X}$ rays during the flare-dip cycle $(\S 3.5 .2$; see also Rodríguez et al. 2001).

\subsubsection{Radio and Infrared}

The source showed weak flares in the radio and infrared bands associated with the soft Xray oscillations on April 17 and 20 (Figure 2 and 3). On April 25, a sinusoidal flux variation, which may be regarded as a flare, was found in the combined infrared light curve (Figure 5). We present the details in $\S 3.3$ and discuss the origin of these radio/infrared flares in $\S$ 4.1. The GBI data also show evidence for such faint flares (e.g., on April 19). No major radio flares exceeding $\sim 100 \mathrm{mJy}$ was detected.

The $K^{\prime}$ and $K s$ magnitudes observed dur- 
ing the quasi-steady state is in the range of 13.2-13.6, which is significantly brighter than the faintest level of $K=14-14.5$ ever observed (Chaty et al. 1996; Mirabel et al. 1998). This result indicates that the source was quite active throughout the campaign, even when no apparent flare was observed. Finally, as noticed from Figure 1, the persistent infrared flux levels seemed to correlate with the soft X-ray intensity on time scale of hours (see $\S 3.4 .2$ and $\S 4.2)$.

\subsubsection{Temporal State of the Source}

To summarize, the source was predominantly in the so-called "plateau" or low/hard state (Class $\chi$, or State C, in Belloni et al. 2000) with a flat radio-mm-(infrared) spectrum (see Figure 6) during the campaign. Recently, it is revealed by VLBA that this state is accompanied with compact jets of 10 AU size (Dhawan, Mirabel \& Rodríguez 2000). The source sometimes showed soft Xray flares (oscillation) classified as Class $\alpha$. In particular, before April 20, a rapid, quasi periodic flare-dip cycle was observed, being associated with faint radio and infrared flares.

In the subsequent sections, we present the detailed results of (1) the whole spectral energy distribution (SED) of GRS 1915+105 in the plateau state, (2) the radio and infrared flares, (3) the quasi-steady infrared emission, and (4) the high energy spectra. The last three topics are further discussed in $\S 4$.

\subsection{The Spectral Energy Distribution of GRS 1915+105 in the Plateau State}

In this subsection we present the whole spectral energy distribution of GRS 1915+105 determined from our data, to have the overall idea about emission mechanisms at each wavelength. Here we focus on the spectra in the plateau state (i.e., a quasi-steady state) to avoid complexity with time variability. Note that this state is most commonly observed from this source (Belloni et al. 2000) being accompanied with the AU-scale (steady or variable) jet and is a potentially very important phase to understand the mechanism of superluminal jets because the state appears to be present as a precursor of large ejection events (Foster et al. 1996). Figure 6 shows the energy spectrum of GRS $1915+105$ in $\nu I_{\nu}$ over the wavelength range of 12 orders of magnitudes. They are corrected for an interstellar absorption (or extinction): we assume $A_{J}=$ 7.1 $\pm 0.2, A_{H}=4.1 \pm 0.2$, and $A_{K}=3.0 \pm 0.1$ from Chaty et al. (1996), which values are derived from mm observations and consistent with X-ray observations of the source. The GIS-PCA-HEXTE spectra are calculated from the data of April 22.4-22.6 and the OSSE spectrum is from April 21.0-25.6. (The normalization of the OSSE spectrum is multiplied by a factor of 1.35 to match the GIS one.) Detailed spectral models used to calculate unfolded spectra are described in $\S$ 3.5.2. For the radio and infrared data, we plot the minimum and maximum flux densities obtained during April 21.0-26.0, when no obvious flares were detected. As for the flux density at $3.2 \mathrm{~mm}$, we use the NMA data taken on April 16, 17, and 18, which may contain a contribution of flares.

The emission in the X-ray to Gammaray bands is dominated by Comptonization, which is approximated with a power law with different slopes below $\simeq 6 \mathrm{keV}$ and above $\simeq 50$ $\mathrm{keV}$. In the soft band, there is a contribution of thermal emission from the optically thick accretion disk, which is rather small in the plateau state as widely reported in the liter- 
ature (Belloni et al. 2000; Muno et al. 2001). The estimated contribution of the reflection component and of the disk component (modeled by the Multi-Color Disk (MCD) model, Mitsuda et al. 1984) are plotted separately from the best-fit parameters of Table 4.

The emission in the infrared band has, as we will show it later, at least 3 different components: a blackbody emission from the companion star, a reprocessing of X-rays in outer parts of the accretion disk, and a synchrotron emission from quasi-steady compact jets, whose contributions to the total are estimated at $20-40 \%, 20-30 \%,<60-30 \%$, respectively (see $\S 4.2$ ). In the figure we plot the contribution from the companion star assuming a blackbody temperature of $4800 \mathrm{~K}$ and a $K$ magnitude of 15.0 (Greiner et al. 2001). We can see that a contribution of the multi-color disk component itself whose parameters are determined from the X-ray data is negligible to the observed infrared flux, as far as the standard disk is assumed.

In the radio band, we see a flat spectrum $\left(\alpha \simeq 0\right.$ for $\left.I_{\nu} \propto \nu^{\alpha}\right)$. The emission is most likely attributed to an optically-thick synchrotron radiation from compact, quasisteady jets. Fitting to the mean VLA fluxes at $1.3 \mathrm{~cm}, 3.6 \mathrm{~cm}$, and $6.0 \mathrm{~cm}$ yields $\alpha=$ $-0.03 \pm 0.09,0.04 \pm 0.03$, and $-0.12 \pm 0.07$, on April 21, 22, and 23, respectively. The best-fit power law spectrum for the April 22 data, simultaneous ones to the presented X-ray spectrum, is plotted in Figure 6. The extrapolation of this spectrum toward shorter wavelengths roughly agrees with the third component of the infrared emission (see above). This is consistent that the flat synchrotron spectrum continues, at least, till the $K$ band $\left(1.4 \times 10^{5} \mathrm{GHz}\right)$ (Ogley et al. 2000; Fender 2001). The absolute radio fluxes showed daily variations, at least by a factor of 3 , indicating different jet activities on a time scale of a day but stable on a time scale of hours.

It would be interesting to compare the SED of GRS 1915+105, a microquasar, with those of quasars. According to typical SEDs of AGNs presented by Elvis et al. (1994) in their Figure 1 and Figure 10, the ratio of $\nu I_{\nu}$ between the radio $\left(\nu=10^{10} \mathrm{~Hz}\right)$ and $\mathrm{X}$ ray $\left(10^{18} \mathrm{~Hz}\right)$ bands is roughly $\sim 10^{-2}$ for radio-loud quasars and $\sim 10^{-5}$ for radio-quiet quasars. (If we use fluxes in UV bands instead of X-rays as a measure of accretion power, where an optically-thick thermal emission from the accretion disk has a peak, the ratio becomes $\sim 10^{-3}$ and $\sim 10^{-6}$, respectively.) On the other hand, Figure 6 shows that the radio-to-X-ray ratio is about $10^{-7}$ for GRS $1915+105$ in the plateau state (and at most $10^{-5}$ even when large radio flares reaching $\sim 1$ Jy were observed). This indicates GRS 1915+105 should fall in the regime of "radio-quiet" microquasars. The flat radio spectrum in the plateau state resembles that of radio-quiet quasars below $\sim 10^{11} \mathrm{~Hz}$. However, in the case of GRS $1915+105$ the flat spectrum seems to continue till $10^{14} \mathrm{~Hz}$, while radio-quiet quasars have the so-called mmbreak around $10^{12} \mathrm{~Hz}$, above which the spectrum steepens $(\alpha \gtrsim-1)$. The reason is unclear but the difference should be taken into account when applying the same jet model developed for AGNs to GRS 1915+105, as discussed by Fender et al. (2000).

\subsection{Radio and Infrared Flares}

In three epochs we detected faint flares in the radio and infrared bands with a duration of 10-20 minutes, although none of them was simultaneously observed in radio and infrared bands, unfortunately. They are most likely 
attributed to the ejection of jets emitting via synchrotron radiation. We present these results in association with the simultaneous Xray light curves. Detailed discussions on the origin of these events are made in $\S$ 4.1.

Figure 2 shows the VLA light curves and simultaneous X-ray light curves on 2000 April 17 taken with the PCA and GIS. As noted, the radio fluxes were variable on a time scale of about $45 \mathrm{~min}$, with the short wavelength varying earlier and more than the longer wavelengths. This behavior has often been seen previously during the X-ray dip/flare cycles of about 30-40 min period (Pooley \& Fender 1997; Eikenberry et al. 1998; Mirabel et al. 1998) and is probably due to the injection of plasma into the flat-spectrum optically thick AU scale jet in the plateau state. The X-ray counts show flare-dip cycles, which are probably caused by the associated disk instability (e.g., Belloni et al. 1997). Although the time resolution is limited, we see flux enhancement in the NMA $3 \mathrm{~mm}$ data on April 18 and in the GBI $3.6 \mathrm{~cm}$ data on April 19, which could be attributed to similar radio flares. On the other hand, the VLA data on April 21, 22, 23 indicate that the source is weaker and the variability is not much more than the random noise. During these epochs X-ray light curves did not show oscillations. The radio spectrum of April 17 was also "flat" i.e., showing approximately the same radio flux density at 6 , 3.6 and $1.3 \mathrm{~cm}$, at the peak of the variation cycle. This is expected since the source size inferred from the variability ( $\simeq 1$ minute) is small, and thus optically thick. (The optically thin, steep spectrum ejecta, if present at all, would not be resolved by the large beam $\geq 1 "$ ).

Similar faint flares were also detected in the $K^{\prime}$ band on April 20. Figure 3 shows a blow up of the $K^{\prime}$ band light curve at 1 minute resolution, plotted with the $A S C A$ GIS and $R X T E$ PCA counts. The X-ray flares correspond to the same class (Class $\alpha$ ) as April 17, although the whole variability pattern seems slightly different from the previous epoch in the sense that the duration of the dip relative to the flare is longer and that the amplitude of the first peak is more prominent compared with the subsequent peaks. As seen from the figure, four (or five if the beginning of the data is counted) separated infrared flares are detected, each has a peak-to-peak amplitude of about 0.3 magnitudes (about $20 \mathrm{mJy}$ if dereddened). Unfortunately, due to the gaps in the GIS data, we could detect only one peak that marks the beginning of the flare at April 20.07. Nevertheless, the linear rise patterns of the X-ray intensity suggest the presence of such flares between them, supporting one-toone correspondence between the infrared and $\mathrm{X}$-ray flares. For later discussion, we estimate the timing of the flares that were not directly observed. In the last panel of Figure 3, the estimated positions of the first peak in each oscillation phase are marked by the downward arrows with a larger width representing the probable error (those with a smaller one are actually observed peaks). Here we have assumed, based on the variability pattern observed in the GIS and PCA data around this epoch, that (1) the duration of the oscillation phase is 15 minutes, and that (2) the flux level at the bottom of the dip, just following the oscillation phase, can be known by a linear interpolation from those before and/or after the cycle.

Figure 5 shows combined infrared light curves taken at the ESO on April 25, together with the GIS light curve. The first 8 points correspond to the $J$ band data, next 9 ones 
to $H$, and the rest to $K s$. To make it easy to see the variability over the whole period, we add offsets to the $J$ and $H$ magnitudes to plot them at the same level as $K s$, so that the data points are smoothly connected by a linear extrapolation. The light curve suggests the presence of a significant, sinusoidal variability with a period of about 10 minutes with a peak-to-peak amplitude of about 0.5 magnitude. Although, unfortunately, most of the strictly simultaneous epoch is not covered by the GIS, there is no evidence for any X-ray flare around this time within offsets of 20-30 minutes.

\subsection{Quasi-Steady Infrared Emission}

\subsubsection{Infrared Spectra}

The infrared spectra $(1.53-2.52 \mu \mathrm{m})$ taken on 2000 April 22 with a resolution of 600 are shown in Figure 7. The visible lines, as already seen by e.g., Martí et al. (2000) and Greiner et al. (2001) are summarized in Table 3. The presence of He II is consistent with the fact that the source was in a low state on April 22 as seen on the X-ray and radio light curves. We also detect the ${ }^{12} \mathrm{CO}$ and ${ }^{13} \mathrm{CO}$ absorption band heads, as discovered by Greiner et al. (2001). The presence of both He II and ${ }^{12} \mathrm{CO} /{ }^{13} \mathrm{CO}$ absorption band heads being a signature of a low-mass system, this confirms the claims of these authors that the companion star is a K-M III star. The $K$ magnitude of the star is estimated to be 14.0-15.0 (Greiner et al. 2000), which accounts for about $20-40 \%$ of the total magnitude observed in the plateau state.

\subsubsection{Correlation between Infrared and $X$ - rays Fluxes in the Plateau State}

In carefully comparing the GIS and infrared $K^{\prime}$ light curves in Figure 1, one may notice a good correlation between the quasisteady flux levels in the two bands, except for the data of April 20, where flares were observed. Such correlation is clearly seen also from the data on April 25 alone, where both soft X-ray and infrared fluxes gradually decreased with similar profiles. Figure 8 shows the correlation between the $K^{\prime}$ magnitude, taken at the Calar Alto observatory, and the corresponding GIS count rates (0.7-10 keV) using exactly simultaneous exposures. Different symbols correspond to different days. Sixteen data points are merged into one point for the $K^{\prime}$ magnitude, thus giving averaged fluxes with a time resolution of about 16 minutes. We do not include the $K s$ data taken at the ESO, for which available simultaneous GIS data are limited, to avoid systematic errors by instrumental effects.

Figure 8 demonstrates the good correlation between them except for the data on April 24. Such correlation can be most easily explained in terms of reprocessing of irradiating X-rays. The reprocessor cannot be located far away from the black hole by larger than $\sim 10^{13} \mathrm{~cm}$ because the time delay is less than $\sim 10$ minutes as seen from the April 25 data, and hence it is most likely the outer parts of the accretion disk. We discuss this correlation quantitatively in $\S 4.2$. The reason for the deviation of the April 24 result is not clear at present.

\subsection{High Energy Spectra}

\subsubsection{OSSE Spectra}

We analyze the OSSE spectrum, covering the highest energy band from $50 \mathrm{keV}$ to 10 
MeV. As mentioned above, GRS 1915+105 was in active states showing rapid soft X-ray oscillations before 2000 April 21, and then became more stable. To see if there is any difference of the high energy spectra between before and after UT $=$ April 21.0, we separately calculated OSSE spectra in the two epochs, from April 18.7 to April 21.0, and from April 21.0 to April 25.6. (It is not feasible to extract the OSSE spectra with the exactly same GTIs as the X-ray data due to limited photon statistics.) Assuming a power law, we find no significant difference in terms of the photon index except that the normalization in the second epoch is larger by about $20 \%$. Hence, we sum the whole data from a total exposure of 169 ks. We find that the whole OSSE spectrum from 0.05 to $\sim 1 \mathrm{MeV}$ can be well described by a single power law $\left(\chi^{2} /\right.$ dof $\left.=5.8 / 16\right)$. We obtained a photon index of $3.12 \pm 0.06$ with a flux of $(2.2 \pm 0.1) \times 10^{-9} \mathrm{ergs} \mathrm{cm}^{-2} \mathrm{~s}^{-1}(0.05$ $10 \mathrm{MeV}$; this value is not corrected for any calibration difference from the other instruments), confirming the results of Zdziarski et al. (2001). The flux is the largest ever observed with OSSE and the photon index is the typical value of this source (Grove et al. 1998; Zdziarski et al. 2001). Figure 9 show the unfolded OSSE spectrum. It is apparent that the data show no evidence of a spectral cutoff. The data appear to suggest a slight hardening of the spectrum above $500 \mathrm{keV}$, but we note that the total significance of the excess over the power law model is less than $1.6 \sigma$. Given the additional systematic uncertainty (not shown in the error bars) in energies above an $\mathrm{MeV}$ (see the discussion in $\S 2.1 .1$ ), we do not consider the excess to be significant, and we make no claim to the existence of any spectral hardening above the best-fit power law. If we combine it with the RXTE HEXTE spec- trum taken on April 22 to cover a wider energy range over $25 \mathrm{keV}-10 \mathrm{MeV}$, we find a spectral break at $56 \pm 8 \mathrm{keV}$, below which the photon index is about $2.67 \pm 0.05$. Note that the presence of the spectral break is confirmed by the HEXTE spectrum alone (Figure 10), which gives a break energy at $42_{-5}^{+12} \mathrm{keV}$, consistent with the above result.

\subsubsection{GIS-PCA-HEXTE Spectra}

In this section, we perform joint spectral analysis from the $A S C A / \mathrm{GIS}, R X T E / \mathrm{PCA}$, and $R X T E /$ HEXTE data, covering the 1-200 $\mathrm{keV}$ band. The detailed analysis of the spectral evolution during the flares is beyond the scope of this paper, and we present the overall spectral properties in typical states. In the joint spectral fitting, we paid special attention to avoid systematic errors. We made cross-calibration between the instruments using the spectra of Crab Nebula and effectively corrected the quantum efficiencies in the response matrices for the slight difference in the obtained best-fit Crab photon index between different instruments, which is turned out to be about 0.05 between GIS and PCA with the use of the latest responses. Absolute normalizations presented in this paper are determined from the GIS (which gives the $2-10 \mathrm{keV}$ Crab flux of $2.2 \times 10^{-8} \mathrm{ergs} \mathrm{cm}^{-2} \mathrm{~s}^{-1}$ ). For all the spectra, we added a $1.5 \%$ systematic error in each energy bin, and conservatively, $3 \%$ for the GIS data below $2.2 \mathrm{keV}$, considering possible uncertainties in the responses for highly absorbed sources. We limited the energy range of the GIS, PCA and HEXTE spectra to $1.2-10 \mathrm{keV}, 3.5-25 \mathrm{keV}$ and $20-200$ $\mathrm{keV}$, respectively.

As mentioned above, during the RXTE observations on April 17 and 20, the source showed a peculiar pattern of variability, char- 
acterized by a rapid oscillation lasting for typically $500-1000 \mathrm{sec}$, followed by a decline of $\sim 200 \mathrm{sec}$ and a slow rise of $\sim 1000$ 2000 sec. The soft X-ray intensity and the hardness have correlations: when the intensity is high, the spectrum becomes hard in the $0.7-10 \mathrm{keV}$ range but soft in the $3-31$ $\mathrm{keV}$ range. We here define four characteristic states: (State I) = the "oscillation high" state, when the soft X-ray flux is larger than roughly the mean value during the oscillation phase (>1800 $\mathrm{c} \mathrm{s}^{-1}$ per PCU in the $16 \mathrm{sec}$ resolution light curve), (State II) = the "oscillation low" state $\left(<1800 \mathrm{c} \mathrm{s}^{-1}\right.$ per PCU), $($ State III $)=$ the "dip" state, when the intensity increases linearly with time between oscillations, and (State IV) = the plateau state, observed after 2000 April 22.

We create the spectra separately for the 4 states defined above, although the $A S C A$ and $R X T E$ data are not strictly simultaneous: for State I, II and III, we use the RXTE data from the April 17 observation and the GIS data taken between April 17.51 and 18.90. For State IV, we calculate them separately for April 22 and 23 from each epoch covered by $R X T E$ but mainly present the results from the April 22 data unless otherwise mentioned. For modeling of the continuum, we use the multi-color disk model plus a hard component whose form is described below, modified with the interstellar absorption. Based on the analysis of frequency-resolved energy spectrum at frequencies higher than several $\mathrm{Hz}$ done by Yamaoka (2000), we adopt a phenomenological model for the hard component, a broken power law with a break energy at 6 $7 \mathrm{keV}$ below which the photon index is fixed at 1.65, multiplied by the high energy cutoff ("highecut" in the XSPEC package). The cutoff is necessary to account for the spectral break (or curvature) around $50 \mathrm{keV}$ as mentioned above. Note that this approximation is only valid for spectral fit in the range below $\sim 200 \mathrm{keV}$ because no cutoff is present at higher energies as we have shown above.

Figure 10 shows unfolded spectra for each state. We find that the fit with this continuum model alone is acceptable for State I and II but not for State III and IV $\left(\chi^{2} /\right.$ dof $=174 / 154$, $178 / 154,532 / 154$, and 794/154, respectively). Nevertheless, the residuals indicates the continuum is reproduced within a $5 \%$ level with this model for any state, which is sufficient to see the difference of the continuum from these unfolded spectra. As noticed from the figure, the spectra in the dip (State III) and plateau (IV) states are very similar to each other and the contribution of the soft, disk component seems much weaker than in the oscillation states (State I and II). In contrast, the difference of the hard band spectrum above $\sim 30$ $\mathrm{keV}$ is not conspicuous between these states, except for a slight difference in the normalization. We can see that a canonical "MCD plus power law" model cannot be used to reproduce the continuum appropriately at least for State III and IV, because the soft X-ray emission below $\sim 6 \mathrm{keV}$ is weaker than the extrapolation of a single power law determined in higher energy bands.

In the spectra of State III and IV, we notice a broad absorption-edge like structure above $\sim 7 \mathrm{keV}$ and a hump around $25 \mathrm{keV}$, which are most likely due to the presence of a reflection component. This is already discussed in Zdziarski et al. (2001). Alternatively, such feature might be explained by a partial covering. In our case, however, the derived column densities $\left(N_{\mathrm{H}} \simeq 3 \times 10^{24} \mathrm{~cm}^{-2}\right)$ exceed one Thomson optical depth, which would lead to a significant increase of the intrinsic lu- 
minosity corrected for Thomson scattering, for example $>2 \times 10^{39} \mathrm{erg} \mathrm{s}^{-1}$ in State III. This is above the Eddington luminosity of the $14 \mathrm{M}_{\odot}$ black hole, and hence we think the "pure" partial covering case is unlikely. We finally find that the combined GIS-PCAHEXTE spectra in these states can be fit with a sum of the MCD model, a broken power law modified with the high energy cut, and a reflection component. In addition, an absorption edge is required at $9.3 \mathrm{keV}$; this is expected when $\mathrm{H}$-like iron ions are present in the line of sight, as indicated by the iron$\mathrm{K}$ absorption line at $7.0 \mathrm{keV}$ in the Chandra HETGS data taken at a quasi simultaneous epoch (Lee et al. 2002). For the calculation of the reflection component, we use the "pexriv" code by Magdziarz \& Zdziarski (1995), approximating the incident spectrum by a cutoff power law model with the parameters determined from the observed spectrum for each state. Since the reflection component should be accompanied by an iron-K emission line, we include it in the fit, assuming a narrow one fixing its energy at $6.5 \mathrm{keV}$. The H-like iron-K absorption line is also included with the same parameters as Lee et al. (2002), which has an equivalent width of about $20 \mathrm{eV}$.

Table 4 summarizes the best-fit parameters in each state (we do not include the reflection component or edge structure at $9.3 \mathrm{keV}$ in State I or II since they are not required). The best-fit model for the spectra on April 20 (=State IV) are plotted in Figure 6. We obtain the innermost radius of the MCD component to be $30 \pm 2 \mathrm{~km}, 32 \pm 2 \mathrm{~km}, 52 \pm 3 \mathrm{~km}$ and $61 \pm 7 \mathrm{~km}$ on April $22(49 \pm 8 \mathrm{~km}$ on April 23) for State I, II, III, and IV, respectively, assuming the distance of $11 \mathrm{kpc}$ and inclination of $66^{\circ}$. Note that these values may be referred only for relative estimates for the disk radius between different states, since the absolute radius obtained from the fit strongly depends on the modeling of the hard component in the soft-energy range, which is highly uncertain.

\section{Discussion}

\subsection{Origin of the Radio and Infrared Flares}

\subsubsection{A Brief Summary of Radio/Infrared Flares Previously Reported from GRS $1915+105$}

From GRS $1915+105$ at least 3 classes of jets have been observed so far according to the classification by Eikenberry et al. (2000): (Class-A) major ejection events with radio fluxes of $\sim 1$ Jy (Mirabel \& Rodríguez 1994; Fender et al. 1999), (Class-B) repeated flares with a period of 30-60 minutes with (dereddened) amplitudes of 100-200 mJy, associated with the X-ray hard-dip/soft-flare cycle (e.g., Mirabel et al. 1998; Eikenberry et al. 1998), and (Class-C) faint infrared flares with amplitudes of $\sim 10$ mJy observed in the softdip/soft-flare cycle (Eikenberry et al. 2000; see also Feroci et al. 1999). Interestingly, the infrared peak of these Class-C events appeared to precede the X-ray dips by 200-600 sec (unless there were time delays of longer than $2000 \mathrm{sec}$ that varied flare by flare). Accordingly they suggest "outside-in" origins for the disk-jet connection. Note that the above classification is only phenomenological at present and distinction between Class-B and Class- $\mathrm{C}$ jets is sometimes ambiguous particularly when X-ray data are not available.

For Class-B jets observed in the $\beta$ state (Belloni et al. 2000), many authors consider that they are triggered by a "spike" in the soft $\mathrm{X}$-ray light curve separating the hard-dip and 
soft-dip phases (e.g., Mirabel et al. 1998; Yadav 2001). The similar decay times between the radio and infrared bands indicates that the adiabatic cooling dominates the radiative cooling (Fender et al. 1997; Fender \& Pooley 1998). As a result, intrinsic power of jets is estimated to be much larger than that observed as radiation (by $\geq 0.05^{-1}$, Fender 2001). The frequency-dependent peak delay of the flare peaks (higher frequencies peak earlier) can be explained in terms of expanding plasmoids radiating through synchrotron emission (e.g., Mirabel et al. 1998). However, as argued by several authors (e.g., Fender \& Pooley 1998), the flat spectra of their peak fluxes in the radio to $K$-band is difficult to explain by a simple "van der Laan" model (van der Laan 1966; Mirabel et al. 1998), rather favoring the partially self-absorbed conical jet models applied for AGNs (Blandford and Königl 1979). More updated models for the quasi-steady jets are proposed by e.g., Falcke \& Biermann (1999) and Kaiser, Sunyaev, \& Spruit (2000).

\subsubsection{Interpretation of Our Results}

The flares observed on April 17 by VLA, in particular at $1.3 \mathrm{~cm}$, and those in the $K^{\prime}$ band on April 20 are very similar in their amplitudes, time profiles, and associated Xray state (Class $\alpha$ ). This tempts us to consider that these flares are essentially the same class of events. The (dereddened) amplitudes, about 10-20 mJy, may suggest their classification as Class-C jets in terms of the flux. The fast rise time, typically $\sim 1 \mathrm{~min}$, constrains the size of the emitting region to be less than $\sim 10^{12} \mathrm{~cm}$. The decay time scale of the flares (about 10 minutes) is also similar between $\lambda=1.3 \mathrm{~cm}$ and $2.2 \mu \mathrm{m}$, supporting previous claims that the adiabatic cooling is dominant in the frequency range at least below $1.4 \times 10^{5}$
GHz. By assuming a flat spectrum below $1.4 \times 10^{5} \mathrm{GHz}$ with an intensity of $15 \mathrm{mJy}$ and the distance of $11 \mathrm{kpc}$, the power of the jet calculated according to Fender (2001) 20 becomes $L_{\text {jet }} \geq 6 \times 10^{36}\left(\frac{0.05}{\eta}\right) F(\Gamma, i) \mathrm{erg} \mathrm{s}^{-1}$. Here $\eta$ is the ratio of the observed power to the total power, which is estimated at $\leq 0.05$ (Fender \& Pooley 2000), and $F(\Gamma, i)$ is the correction factor for relativistic bulk motion. If we assume the inclination of $i=66^{\circ}$ and the velocity of $0.98 c$ (thus giving $F(\Gamma, i) \simeq 140$ ) measured for the Class-A event of 1997 October/November (Fender et al. 1999), the minimum jet power reaches $8 \times 10^{38} \mathrm{erg} \mathrm{s}^{-1}$. This value amounts to about twice the observed $\mathrm{X}$ ray to Gamma-ray luminosity, $4 \times 10^{38} \mathrm{erg} \mathrm{s}^{-1}$ (1 keV-10 MeV) on April 22.

Timing of the radio/infrared flares with respect to the X-ray variability is important to constrain the mechanism of jets. For the radio flares on April 17, the observed frequencydependent peak delay can be explained by an expansion of a plasmoid or an internal-shock region (see Kaiser, Sunyaev, \& Spruit 2000), which predicts a time delay of $\Delta t \propto \lambda^{2 / 3}$ (van der Laan model for an electron energy distribution of $p \simeq 0$ for $\left.N(E) \propto E^{-p}\right)$ or $\Delta t \propto \lambda^{1}$ (self-similar regime of the Blandford $\&$ Königl jet), respectively. As seen from Figure 2, $\Delta t \sim 5$ minutes between $\lambda=1.3$ and $3.6 \mathrm{~cm}$. In either model, $\Delta t$ at $1.3 \mathrm{~cm}$ is estimated at $\sim 5$ minutes, indicating the start time of each radio flare coincides with the time of the first peak in the X-ray oscillation phase. The timing of radio flares on 1996 October 25 associated with Class $\alpha$ (Figure 5 of Pooley \& Fender 1997) seems consistent with this picture, although their amplitudes, about $50 \mathrm{mJy}$, were about 3 times larger than ours.

${ }^{20}$ In the formula for $L_{\text {jet }}$ given in Section 7 of Fender (2001) $10^{36}$ should be replaced by $1.4 \times 10^{35}$. 
These first X-ray peaks may correspond to the soft X-ray "spike" observed in the $\beta$ state, which separates the states with hard and soft spectra, possibly triggering Class-B jets (e.g., Yadav 2001). Obviously, the duration of the $1.3 \mathrm{~cm}$ flare is shorter than that of the X-ray oscillation phase. This rules out the scenario that the radio flare is a superposition of many short flares of a similar amplitude produced by "every" X-ray peak during the whole oscillation phase.

On the other hand, the interpretation for the infrared flares observed on April 20 is not straightforward. As noticed from Figure 3, the timing of the rise of each infrared flare does not match any of the first peak in X-ray oscillation, which are indicated by the arrows in the third panel. (This argument is firm, at least for the infrared flare on April 20.0820.09 , which is free from the data gap problem.) Therefore, if we relate the timing of infrared flares to the start time of each X-ray oscillations, as done for the April 17 events, two possibilities arise i.e., (1) the infrared flare followed the first peak of X-ray oscillation phase, or (2) the infrared preceded the X-ray (as the case suggested by Eikenberry et al. 2000). In either case, as seen from Figure 3, the time delay seems to be different flare by flare, ranging from $\simeq 5$ to 30 minutes (plus integer times the one cycle period, $\simeq 45$ minutes). We favor the first possibility (infrared delay) because it is more natural to attribute the ejection to change of the state of the accretion disk observed in X-rays and because it seems difficult to find physical mechanisms causing such a long delay in the second case. In the first case, the longest delay time indicates the distance of the infrared emitting region can be far by $5 \times 10^{13} \mathrm{~cm}$ from the black hole assuming the jet velocity of $\sim 0.9 c$. This is consistent with the size of the quasi-steady jet in the plateau state, $2 \mathrm{AU}\left(=3 \times 10^{13} \mathrm{~cm}\right.$, Dhawan et al. 2000). The highly delayed infrared flare can then be understood by an internal shock that occurred far away from the jet base, being triggered by a matter ejected at the beginning of the X-ray oscillation. On the other hand, the size of the "lit-up" region by the internal shock must be $\leq 10^{12} \mathrm{~cm}$ from the constraints of the time variability, which is much smaller than the scale of jet length. This implies that the jet is well collimated with an opening angle of $\lesssim 1 / 10$, which is consistent with the values derived from other observations (e.g., Fender et al. 1999; Chaty et al. 2001).

We consider it unlikely, though cannot rule out, that the ejections occurred at the end of the oscillation i.e., start of the dip. If the first two flares at April 20.08-20.09 and 20.12520.135 had been triggered by the starts of the dip within $\simeq 20$ minutes, the infrared flare at April 20.15 would have occurred before the start of the dip by $\simeq 5$ minutes, contrary to the previous causality. Therefore, in either case where the infrared flares followed or preceded the start of the dip, we have to introduce a long time delay, larger than 45 minutes, to reconcile the timing relation for the event at April 20.15. Finally, there could be even an extreme possibility that the ejections occurred during the dip phase without showing no apparent signature in X-rays. Indeed, the infrared flare-like flux variation detected on April 25 appeared not to be accompanied by any strong X-ray variability, although this is not conclusive due to the data gap. The detection of such variability might be consistent with the picture that the plateau is a quite active state exhibiting continuous internal shocks where a weak perturbation in the jet flow could cause faint radio/infrared flare 
even without a dramatic change in the accretion state observable in X-rays.

In summary, it is not clear whether or not all the faint ( $\lesssim 40$ mJy) radio/infrared flares observed so far indeed belongs to a single class of events. Although one-to-one correspondence between radio/infrared and X-ray variability pattern strongly indicates a tight disk-jet connection, it is not obvious yet what kind of transition seen in X-rays actually triggers the ejections. For example, the events detected by Eikenberry et al. (2000) are associated with the soft-dip/soft-flare cycle, while ours seems to be associated with the transition from the hard-dip to the soft-flare phase, similarly to the case of class-B jets (Mirabel et al. 1998). Even within similar states, as in our observations on April 17 and 20, the timing relation between the radio/infrared flare and X-rays seems to be different. More observations are necessary to reveal the nature of these "faint" flares. They are potentially very important to understand the physics of the quasi-steady jet in the plateau state, considered to exist in many X-ray binary systems (Fender et al. 2000).

\subsection{Origin of the Quasi-Steady Infrared Emission}

Here we summarize possible origins of infrared emission of GRS 1915+105, as listed in e.g., Mirabel et al. (1997): (1) emission from the companion star, (2) reprocessing of $\mathrm{X}$-rays in the outer parts of the accretion disk, (3) optically thin free-free emission from an X-ray driven wind (van Paradijs et al. 1994), (4) synchrotron emission from jets (Sams, Eckard \& Sunyaev 1996), (5) Doppler-shifted line emission from ions in the relativistic jets (Mirabel et al. 1997), and (6) thermal dust reverberation of energetic outbursts (Mirabel et al. 1996). Below we constrain the contribution of the second origin.

As we have shown in Figure 8, we find a good correlation between the flux of the quasisteady infrared emission and that of X-rays. To be quantitative, we fit the relation between the infrared flux $F_{\mathrm{IR}}$ (unit: mJy) dereddened with $A=3.0$ versus the X-ray flux $F_{\mathrm{X}}$ (unit: GIS $\mathrm{c} \mathrm{s}^{-1}$ ) with a linear function in the form of

$$
F_{\mathrm{IR}}=F_{\mathrm{IR} 0}+C \times F_{\mathrm{X}}
$$

based on a simple assumption that the reprocessed infrared flux was proportional to the irradiating X-ray flux and other components were constant. The data of April 24 are not included. The fit is quite good and we obtain the best fit parameters of $F_{\text {IR } 0}=37 \mathrm{mJy}$ and $C=7 \times 10^{-5}$. This indicates that the contribution of the reprocessing of X-rays is about 20-30\% of the total observed magnitude $\left(K^{\prime}\right.$ $=13.25-13.4$ on 2000 April 21-25).

Thus, the contribution from the companion star and from reprocessing of X-rays in outer parts of the accretion disk is likely to account for about $20-40 \%$ and $20-30 \%$ of the observed, quasi-steady $\mathrm{K}$ magnitudes in the plateau state, respectively. The rest $60-30 \%$ of the total infrared flux (i.e., 30-15 mJy) must have other origins than (1), (2) and (6) listed above. As mentioned earlier, if we attribute this to synchrotron radiation from the jets, the result is consistent with the picture that the emission from quasi-steady compact jets continues from the radio to near-infrared bands with a nearly flat spectrum.

\subsection{Origin of the High Energy Spec- tra}

We have presented the energy spectra of GRS1915+105 in the $1 \mathrm{keV}-10 \mathrm{MeV}$ band combining the contemporaneous $A S C A$ GIS, 
RXTE PCA and HEXTE, and CGRO OSSE observations. These data provide the most complete coverage in high energy bands ever presented in the literature for GRS 1915+105 and are used to construct the spectral model (Figure 6). In the 1-200 keV band, using the simultaneous data, we could obtain the energy spectra separately in the three typical states during the soft X-ray flare (Class $\alpha$ ) and in the plateau state (Class $\chi$ ).

Power law spectra with photon indices of $\sim 2.5$ extending above $\sim 1 \mathrm{MeV}$ without cutoff are often observed from black hole candidates (BHCs) in the "soft state" (e.g., Grove et al. 1998; Gierliński et al. 1999), in which most of the energies are emitted below $5 \mathrm{keV}$ via thermal emission from the optically thick accretion disk. These soft-state energy spectra are obviously different from the typical energy spectra in the hard state, which show power-law spectra with photon indices 1.7 and a thermal cutoff around 100200 keV (e.g., Gierliński et al. 1997; Grove et al. 1998). The fact that GRS 1915+105 has a very "soft" $(\Gamma \approx 3)$ spectrum indicate that its spectral state corresponds to the soft state rather than the hard state, although, in the plateau state, a contribution of the disk component seems much weaker than in the soft-state spectra of other BHCs. The absence of high energy cutoff suggests the presence of non-thermal electrons, as Zdziarski et al. (2001) indicate that the spectra of GRS 1915+105 can be explained by Comptonization model with a hybrid plasma with thermal and non-thermal electrons.

The spectral change in the oscillation phase have been reported by many authors, which is considered to be caused by thermal-viscous instability (e.g., Belloni et al. 1997). In fact, as pointed by Yamaoka (2000), the appar- ently high temperature and small radius in the oscillation high state, as confirmed from our observations, can be explained by emission from optically-thick advection dominated accretion flow, so-called a slim-disk (Watarai et al. 2000), as a consequence from such transition. Such transition may trigger ejection of jets observed in radio and infrared bands $(\S 4.1)$.

We found that the spectrum in the dip phase between rapid oscillations and that in the more stable plateau state look similar, confirming that they are essentially the same class of spectral state (State C, Belloni et al. 2000). Indeed, they also share similar timing properties, showing QPOs with harmonics (Rodríguez et al. 2001). The correlation between the QPO frequency and the disk radius in the plateau state between April 22 and 23 is consistent with the claims by previous authors, in the sense that a higher frequency QPO is observed with a smaller disk radius. Such correlation could be explained by the accretion ejection instability (Tagger \& Pellat 1999; Rodríguez et al. 2001).

For State III and IV, we find the solid angle of the reflector subtending the $\mathrm{X}$-ray source is $\Omega / 2 \pi \simeq 0.6-0.9$. This value predicts a total equivalent width of iron-K emission lines of 50-100 eV (e.g., Basko 1978; George \& Fabian 1991), whereas we obtain only $\sim 10 \mathrm{eV}$ from our fits. Also, the Chandra HETGS data also show very small equivalent widths for (narrow) emission lines in the range of 6.4-6.6 $\mathrm{keV}$, which are consistent with our results. Such apparently small line intensities could be explained by significant blurring of the line profile via Doppler redshift and/or Compton scattering, or other physical mechanism such as Auger destruction (Ross, Fabian \& Brandt 1996). Note that the obtained edge depth 
from H-like iron, $\tau \simeq 0.05$, corresponds to the iron column density of $\log N_{\mathrm{Fe} 26} \simeq 18.7$, which is by about one order of magnitudes larger than that derived from a simple curve of growth analysis of the absorption line (Lee et al. 2002). This implies that the resonance absorption lines are partially refilled by the emission line photons at the same energy.

In our observations, we find that the spectrum above $\sim 30 \mathrm{keV}$ does not significantly differ between different states. The spectrum keeps the same slope $(\Gamma \approx 3$ above $50 \mathrm{keV})$, and shows similar fluxes even during the oscillation phase in spite of the large flux variation below $\lesssim 10 \mathrm{keV}$. The eight OSSE observations summarized by Zdziarski et al. (2001) also show that, on much longer time scales, the power law slope above $50 \mathrm{keV}$ is almost always constant around $\Gamma \simeq 3.0$ and the flux stays within factor of 2 except for one occasion. Thus, the constant slope and the lack of correlation with soft X-ray states may be persistent features of the hard X-ray spectrum of GRS 1915+105. The stable emission above $\sim 50 \mathrm{keV}$ implies that the energy distribution of high-energy electrons responsible for Comptonization is regulated by some (unknown) mechanism, not being affected by the change of the state in the accretion disk. These results give strong constraints for theoretical modeling of the accretion flow in GRS $1915+105$.

\section{Conclusion}

We have performed a multiwavelength campaign of GRS 1915+105 from 2000 April 16 to 25 , covering the wide energy band in radio $(\lambda=13.3-0.3 \mathrm{~cm})$, near-infrared $(J, H$, $K)$, X-rays and Gamma-rays (from $1 \mathrm{keV}$ to $10 \mathrm{MeV}$ ), with $A S C A, R X T E, B$ eppoSAX, $C G R O$, and ground facilities including the
VLA, the NMA, the ESO, and the Calar Alto Observatory. This is one of the largest coordinated observations ever performed for this source. Main results obtained from this campaign are summarized as follows.

(1) The source was predominantly in the "plateau" state (Class $\chi$ ) and sometimes showed soft X-ray flares (oscillation) classified as Class $\alpha$. Before April 20.3, a rapid, quasi periodic flare-dip cycle was observed, being associated with faint radio and infrared flares.

(2) The spectral energy distribution of GRS $1915+105$ in the plateau state is presented covering the energy range of 12 orders of magnitudes. The radio to near-infrared spectra are flat, consistent with the presence of optically-thick compact jet. The X-ray to Gamma-ray emission is dominated by Comptonization.

(3) In three epochs we detected faint flares in the radio or infrared bands with amplitudes of 10-20 mJy. The radio flares observed on April 17 shows frequency-dependent peak delay, consistent with an expansion of synchrotronemitting region starting at the transition from the hard-dip to the soft-flare states in X-rays. On the other hand, the infrared flares on April 20 appear to follow (or precede) the beginning of X-ray oscillations with an inconstant time delay of $\simeq 5-30 \mathrm{~min}$. This implies that the infrared emitting region, probably the place of an internal shock in the quasi-steady jet, is located far from the black hole by $\gtrsim 10^{13} \mathrm{~cm}$, while its size is $\lesssim 10^{12} \mathrm{~cm}$ constrained from the time variability.

(4) A good correlation is found between the quasi-steady flux level in the near-infrared band and that in the X-ray band after 2000 April 21. This indicates that a part of the observed infrared emission is attributable to reprocessing of X-rays, probably in outer parts 
of the accretion disk. The contribution from the companion star and from reprocessing of X-rays accounts for about $20-40 \%$ and 20 $30 \%$, respectively, of the quasi-steady $K$ magnitude $\left(K^{\prime}=13.25-13.4\right)$ in the plateau state.

(5) The time averaged OSSE spectrum from $50 \mathrm{keV}$ to $\sim 1 \mathrm{MeV}$ is represented by a single power law with a photon index of 3.1 with no significant high energy cutoff, confirming the results of Zdziarski et al. (2001). It did not show significant difference, except for the normalization, between before and after April 21.0 when the rapid soft X-ray flares ceased. The continuum in the $1-200 \mathrm{keV}$ band can be modeled by a broken power law with a break energy at 6-7 keV, modified with a high energy cutoff, plus the multi-color disk model. In addition, we significantly detect a reflection component from a cold (or slightly warm) matter with a solid angle of $\Omega / 2 \pi=0.6-1.0$ in the spectra of the dip and plateau states. The power-law slope above $\sim 30 \mathrm{keV}$ is found be very similar between different states in spite of large flux variations in soft X-rays, implying that the energy distribution of high energy electrons responsible for Comptonization is regulated by some (unknown) mechanism, not being affected by the change of the state in the accretion disk.

We thank Neil Gehrels for making the OSSE observation possible coordinated with the campaign and Robert Fender for discussion. S.C. is very grateful to the ESO/NTT team, and especially Leonardo Vanzi, for all the high quality service observations performed every day during one week of this multiwavelength campaign, as a target of opportunity. S.C. also acknowledges support from grant F/00-180/A from the Leverhulme Trust. We also thank Kazuhiro Sekiguchi (Subaru tele- scope) and Taichi Kato (variable star network) for their efforts and help in organizing the campaign. This research has made use of the data of the Green Bank Interferometer (GBI), which is a facility of the National Science Foundation operated by the NRAO in support of NASA High Energy Astrophysics programs. 


\section{REFERENCES}

Basko, M.M. 1978, ApJ, 223, 268

Belloni, T., Méndez, M., King, A.R., van der Klis, M., van Paradijs, J. 1997, ApJ, 479, 145

Belloni, T., Klein-Wolt, M., Mendez, M., van der Klis, M., \& van Paradijs, J., 2000, A\&A, 355, 271

Blandford, R.D. \& Königl, A. 1979, ApJ, 232, 34

Boella, G., Butler, R.C., Perola, G.C., Piro, L., Scarsi, L., \& Bleeker, J.A.M., 1997a, A\&AS, 122, 299

Boella, G. et al. 1997b, A\&AS, 122, 327

Castro-Tirado, A.J., Brandt, S., \& Lund, N. 1992, IAU Circ., 5590

Castro-Tirado, A.J., et al. 1994, ApJS 92, 469

Castro-Tirado, A.J., Geballe, T.R., \& Lund, N. 1996, ApJ, 461, L99

Chaty, S., Mirabel, I.F., Duc, P.A., Wink, J.E., \& Rodríguez, L.F. 1996, A\&A 310, 825

Chaty, S. et al. 2001, A\&A, 366, 1035

Dhawan, V., Gross, W.M., \& Rodríguez, L.F. 2000, ApJ, 540, 863

Dhawan, V., Mirabel, I.F., \& Rodríguez, L.F. 2000, ApJ, 543, 373

Elvis, M. et al. 1994, ApJS, 95, 1

Eikenberry, S.S., Matthews K., Morgan E.H., Remillard, R.A., \& Nelson, R.W. 1998, ApJ, 494, L61
Eikenberry, S.S. et al. 2000, ApJ, 532, L33

Falcke, H. \& Biermann, P.L. 1999, A\&A, 342, 49

Fender, R.P., Pooley, G.G., Brocksopp, G., \& Newell S.J. 1997, MNRAS, 290, L65

Fender, R.P. \& Pooley, G.G. 1998, MNRAS, 300,537

Fender, R.P. et al. 1999, MNRAS, 304, 865

Fender, R.P., Pooley G.G., Durouchoux, P., Tilanus, R.P.J., \& Brocksopp, C. 2000, MNRAS, 312, 853

Fender, R.P. \& Pooley, G.G. 2000, MNRAS, 318, L1

Fender, R.P. 2001, MNRAS, 322, 31

Feroci, M., Matt G., Pooley, G., Costa, E., Tavani, M., \& Belloni, T. 1999, A\&A 351, 985

Feroci, M. et al. 2001, in preparation

Fiore, F., Guainazzi, M., \& Grandi, P., 1999, Cookbook for BeppoSAX NFI Spectral Analysis

Fishman, G.J., et al. 1989, in Proc. of Gamma-Ray Observatory Science Workshop, ed. N. Johnson (Greenbelt: Goddard Space Flight Center), 2-39

Foster, R.S. et al. 1996, ApJ, 467, L81

Frontera, F., Costa, E., Dal Fiume, D., Feroci, M., Nicastro, M., Orlandini, M., Palazzi, E. \& Zavattini, G., 1997, A\&AS, 122,357

George I.M. \& Fabian A.C. 1991, MNRAS, 249, 352 
Gierliński, M., et al. 1997, MNRAS, 288, 958

Gierliński, M., Zdziarski, A.A., Pouranen, J., Coppi, P.S., Ebisawa, K., \& Johnson, W.N. 1999, MNRAS, 309, 496

Greiner, J., Cuby, J.G., McCaughrean, M.J., Castro-Tirado, A.J., Mennickent, R.E., 2001, A\&A, 373, L37

Greiner, J., Cuby, J.G., \& McCaughrean, M.J., 2001, Nature, 414, 522

Grove, J.E., Johnson, W.N., Kroeger, R.A., McNaron-Brown, K., \& Skibo, J.G. 1998, ApJ 500, 899

Hannikainen et al. 2001, Astrophysics and Space Science, 276, 45

Harmon, B.A., et al. 1992, in Proc. Compton Observatory Science Workshop, ed. C.R. Shrader, N. Gehrels, and B. Dennis (Washington: NASA) 69

Jahoda K., et al. 1996, SPIE 2808, 59

Johnson, W.N., et al. 1993, ApJS, 86, 693

Kaiser, C.R., Sunyaev, R., \& Spruit, H.C. 2000, A\&A, 356, 975

Lee, J.C.,

Reynolds, C.S., Schulz, N.S., Remillard, R., Blackman, E.G., Fabian, A.C. 2002, ApJ, in press (astro-ph/0111132)

Magdziarz, P., \& Zdziarski, A.A. 1995, MNRAS, 273, 837

Martí, J., Mirabel, I. F., Chaty, S., Rodríguez, L. F. 2000, A\&A, 356, 943

Mirabel, I.F. \& Rodríguez, L.F. 1994, Nature, 371,46
Mirabel, I.F. et al. 1996, ApJ, 472, L111

Mirabel, I.F., Bandyopadhyay, R, Charles, P.A., Shahbaz, T., \& Rodríguez, L.F. 1997, ApJ 477, L45

Mirabel, I.F. et al. 1998, A\&A, 330, L9

Mirabel, I.F. \& Rodríguez, L.F. 1999, ARAA, 37,409

Mitsuda K., et al. 1984, PASJ, 36, 7

Muno, M.P. et al. 2001, ApJ, 556, 515

Ogley, R.N., Bell Burnell, S.J., Fender, R.P., Pooley, G.G., \& Waltman, E.B. 2000, MNRAS, 317, 158

Ohashi, T. et al. 1996, PASJ, 48, 157

Okumura, S. K. et al. 2000, PASJ, 52, 393

Orosz, J.A. et al. 2001, ApJ, 555, 489

Persson, S.E., Murphy, D.C., Krzeminski, W., Roth, M., \& Rieke, M.J. 1998, AJ, 116, 2475

Pooley, G.G. \& Fender R.P. 1997, MNRAS, 292, 925

Rodríguez, L.F. et al. 1995, ApJ, 101, 173

Rodríguez, J., Varniere, P., Tagger, M., \& Durouchoux, P. 2001, Astrophysics and Space Science, 276, 235

Rodríguez, J., Durouchoux, P., Mirabel, I.F., Ueda, Y., Tagger, M., \& Yamaoka, K. 2001, submitted to A\&A

Ross, R.R., Fabian, A.C., Brandt, W.N. 1996, MNRAS, 278, 1082

Rothschild, R.E., et al. 1998, ApJ 496, 538 
Sams, B.J., Eckart, A., \& Sunyaev, R. 1996, Nature, 382, 47

Sánchez-Fernández, C., 2001, thesis, Madrid

Tagger, M. \& Pellat, R. 1999, A\&A, 349, 1003

Tanaka, Y., Inoue, H., \& Holt, S. S. 1994, PASJ, 46, L37

Tsutsumi, T., Morita, K. I., \& Umeyama, S. 1997, in Astronomical Data Analysis Software and Systems VII, eds. G Hunt and H. E. Payne (ASP, San Francisco), 50

van der Laan, H. 1966, Nature, 211, 1131

van Paradijs, J., Telesco, C.M., Kouveliotou, C., \& Fishman, G.J. 1994, ApJ 429, L19

Watarai, K., Fukue, J., Takeuchi, M., Mineshige, S. 2000, PASJ, 52, 133

Yadav, J.S. 2001, ApJ, 548, 876

Yamaoka, K. 2000, thesis, Univ. of Tokyo

Yamashita, A., Dotani, T., Ezuka, H., Kawasaki, M., Takahashi, K. 1999, Nucl. Insr. and Meth. A 436, 68

Zdziarski, A.A., Grove, J.E., Poutanen, J., Rao, A.R., \& Vadawale, S.V. 2001, ApJ 554, L45

This 2-column preprint was prepared with the AAS LATEX macros v4.0. 
Fig. 1. - The whole multiwavelength light curves of GRS 1915+105 obtained from the 2000 April campaign, covering between April 16 and 25. They are sorted in energy band (higher energy in upper). From upper to lower panels, CGRO OSSE (150-300 $\mathrm{keV}$ : unit Crab), OSSE (50-150 keV: Crab), CGRO BATSE (20-100 keV: photons $\mathrm{cm}^{-2}$ $\mathrm{s}^{-1}$ ), BeppoSAX PDS (15-300 keV: c s$^{-1}$ ), RXTE HEXTE (15-120 keV: $\mathrm{c} \mathrm{s}^{-1}$, sum of cluster-0 and -1), RXTE PCA (4-25 keV: c $\mathrm{s}^{-1}$, sum of PCU0, 2 and 3 ; the data when PCU3 was off are not plotted), RXTE ASM (1.5-12 keV: c s$\left.^{-1}\right)$, BeppoSAX MECS (1.5$\left.10 \mathrm{keV}: \mathrm{c} \mathrm{s}^{-1}\right), A S C A$ GIS (0.7-10 keV: $\mathrm{c} \mathrm{s}^{-1}$, sum of GIS2 and GIS3), near-infrared $J$ - mag (ESO), $H$-mag (ESO), $K s$ - mag (ESO), $K^{\prime}-$ mag (Calar Alto), NMA $(\lambda=3.2 \mathrm{~mm}: \mathrm{mJy})$, VLA (1.3 cm: mJy), VLA (3.6 cm: mJy), VLA (6.0 cm: mJy), GBI (3.6 cm: mJy), and GBI (13.3 cm: mJy). The arrows in the GIS panel indicate the times of the first peak of soft X-ray flares seen in the GIS medium bitrate data.

Fig. 2.- Multiwavelength light curves of GRS $1915+105$ on 2000 April 17.53-17.65. From upper to lower panels: RXTE PCA at 16 s resolution $\left(4-25 \mathrm{keV}\right.$ : unit $\left.\mathrm{c} \mathrm{s}^{-1}\right)$, the PCA hardness ratio $4-10 \mathrm{keV}$ and $10-25 \mathrm{keV}$, $A S C A$ GIS at $4 \mathrm{~s}$ resolution $(0.7-10 \mathrm{keV}$ : c $\left.\mathrm{s}^{-1}\right)$, and radio fluxes by VLA $(1.3 \mathrm{~cm}, 3,6$ $\mathrm{cm}$, and $6.0 \mathrm{~cm}: \mathrm{mJy})$.

Fig. 3.- Multiwavelength light curves of GRS $1915+105$ on 2000 April 19.95-20.25. From upper to lower panels: RXTE PCA at 16 s resolution $\left(4-25 \mathrm{keV}\right.$ : unit $\left.\mathrm{c} \mathrm{s}^{-1}\right), A S C A$ GIS at 4 s resolution (0.7-10 keV: $\left.\mathrm{c} \mathrm{s}^{-1}\right)$, and infrared $K^{\prime}$ magnitude taken at the Calar Alto observatory. The arrows with a large (small) width in the bottom panel indicate the estimated (observed) time of the first peak of the soft X-ray flares ( $\operatorname{see} \S 3.3$ ).

Fig. 4. - Multiwavelength light curves of GRS $1915+105$ on 2000 April 23.78-23.83. From upper to lower panels: BeppoSAX PDS at $4 \mathrm{~s}$ resolution $\left(15-300 \mathrm{keV}\right.$ : unit $\left.\mathrm{c} \mathrm{s}^{-1}\right)$ and MECS (1.5-10 keV).

Fig. 5.- Multiwavelength light curves of GRS $1915+105$ on 2000 April 25.35-25.41. From upper to lower panels: $A S C A$ GIS at 4 s resolution (0.7-10 keV: unit $\left.\mathrm{c} \mathrm{s}^{-1}\right)$ and nearinfrared magnitudes $(J, H$, and $K s)$ taken at the ESO. Offsets of -4.6294 and -1.9179 are added to the $J$ and $H$ magnitudes, respectively.

Fig. 6. - The spectral energy distribution of GRS $1915+105$ in the plateau state (except for the $94 \mathrm{GHz}$ data) obtained from the campaign. Interstellar absorption and extinction are corrected. The $1-200 \mathrm{keV}$ data are calculated from the simultaneous GISPCA-HEXTE spectra on April 22.4-22.6 and the $0.05-10 \mathrm{MeV}$ data are from the averaged OSSE spectrum on April 21.0-26.0. The near-infrared data correspond to the minimum and maximum flux densities during April 21.0-26.0 observed at the ESO. The three fluxes in the radio band $(5,8$, and 22 $\mathrm{GHz}$ ) are taken from the VLA data on April 21,22 , and 23 , while those at $94 \mathrm{GHz}(\lambda=3.2$ $\mathrm{mm}$ ) are from the NMA data on April 16, 17, and 18. Models from right to left: the long dashed line corresponds to an estimated contribution of the reflection component $(\Omega / 2 \pi$ $=0.64, \xi=2$; see Table 4 for detailed explanation) with an iron-K emission line (at $6.5 \mathrm{keV}$ with an equivalent width of $4 \mathrm{eV}$ ), the solid line the multi-color disk $\left(k T_{\text {in }}=\right.$ $\left.0.70 \mathrm{keV}, R_{\text {in }}=61 \mathrm{~km}\right)$, short-dashed line 
(right) the blackbody spectrum of the companion star with a temperature of $4800 \mathrm{~K}$, and the short-dashed straight line (left) the best-fit power law determined from the 3 radio fluxes on April $22\left(I_{\nu} \propto \nu^{0.04}\right)$, which are simultaneous data to the $1-200 \mathrm{keV}$ spectra plotted here.

Fig. 7.- The infrared spectra of GRS 1915+105 taken at the ESO on 2000 April 22 at a resolution of $\lambda / \Delta \lambda=600$ (upper: the 1.53-1.80 $\mu \mathrm{m}$ range, lower: 2.03-2.55 $\mu \mathrm{m}$ ). The spectra are normalized and therefore vertical units are arbitrary.

Fig. 8.- The correlation between the infrared $K^{\prime}$ magnitude (taken at Calar Alto) and the GIS count rates $(0.7-10 \mathrm{keV})$, each averaged for $\sim 16$ minutes with exactly simultaneous exposures. Different symbols correspond to different days (open circles: April 21, diamonds: April 22, open squares: April 24, filled circles: April 25). The data of April 20 are not included, which showed infrared flares.

Fig. 9.- The OSSE unfolded spectrum in the $0.05-10 \mathrm{MeV}$ range taken during 2000 April 18.7 to 25.6. The model is the best fit power law with a photon index of 3.12.

Fig. 10. - The combined GIS-PCA-HEXTE unfolded spectra in the $1-200 \mathrm{keV}$ band in the four different states (see text), (I) the oscillation-high, (II) the oscillation-low, (III) the dip, and (IV) the plateau state, each multiplied by a factor of $8,4,2$, and 1 , respectively, for clarity of the plots. 
TABLE 1

Log of the Multiwavelength OBservations

\begin{tabular}{|c|c|c|c|}
\hline Observatory/Telescope & Instrument & Band & Observation Date (UT, 2000 Apr) \\
\hline$C G R O$ & $\begin{array}{l}\text { OSSE } \\
\text { BATSE }\end{array}$ & $\begin{array}{l}50 \mathrm{keV}-10 \mathrm{MeV} \\
20-100 \mathrm{keV}\end{array}$ & $\begin{array}{l}18.7-25.6 \\
\text { monitor }\end{array}$ \\
\hline $\begin{array}{l}\text { BeppoSAX } \\
\cdots\end{array}$ & $\begin{array}{l}\text { PDS } \\
\text { MECS/LECS }\end{array}$ & $\begin{array}{l}15-300 \mathrm{keV} \\
0.1-10 \mathrm{keV}\end{array}$ & $\begin{array}{l}15.80-22.89,23.35-23.89,24.34-24.48 \\
\text { same as above }\end{array}$ \\
\hline$R X T E$ & HEXTE & $15-250 \mathrm{keV}$ & $\begin{array}{l}17.54-17.64,20.20-20.36,22.39-22.55,23.32-23.42,24.05-24.41 \\
24.78-24.82,25.18-25.26,25.85-25.88\end{array}$ \\
\hline$\ldots$ & PCA & $2-60 \mathrm{keV}$ & same as above \\
\hline$\cdots$ & ASM & $1.5-12 \mathrm{keV}$ & monitor \\
\hline$A S C A$ & GIS/SIS & $0.5-10 \mathrm{keV}$ & $17.57-25.50$ \\
\hline $\begin{array}{l}\mathrm{ESO} / \mathrm{NTT} \\
\cdots \\
\cdots\end{array}$ & $\begin{array}{l}\text { SOFI (Imaging) } \\
\text { SOFI (Imaging) } \\
\text { SOFI (Spectroscopy) }\end{array}$ & $\begin{array}{l}J-H-K s \\
K s \\
1.53-2.52 \mu \mathrm{m}\end{array}$ & $\begin{array}{l}19.35-19.37,20.39-20.43,21.34-21.36,25.37-25.39 \\
23.34-23.40,24.35-24.40 \\
22.3\end{array}$ \\
\hline Calar Alto / 1.23m & MAGIC & $K^{\prime}$ & $20.06-20.19,21.07-21.21,22.08-22.21,24.03-24.20,25.05-25.21$ \\
\hline NMA & & $88.6,100.6 \mathrm{GHz}$ & $16.88-17.01,17.76-17.94,18.72-18.81$ \\
\hline VLA & & $5,8,22 \mathrm{GHz}$ & $17.54-17.64,21.53-21.58,22.35-22.54,23.33-23.45$ \\
\hline GBI & & $2.25,8.3 \mathrm{GHz}$ & monitor (except 21-24) \\
\hline
\end{tabular}


TABLE 2

Averaged Near-Infrared Magnitudes of GRS 1915+105 Observed at the ESO

\begin{tabular}{lccc}
\hline \hline Date (UT, 2000 Apr) & $J$ & $H$ & $K s$ \\
\hline $19.35-19.38$ & $17.612 \pm 0.034$ & $14.856 \pm 0.012$ & $13.385 \pm 0.054$ \\
$20.39-20.43$ & $17.586 \pm 0.037$ & $14.872 \pm 0.032$ & $13.396 \pm 0.063$ \\
$21.33-21.36$ & $17.707 \pm 0.025$ & $14.900 \pm 0.022$ & $13.484 \pm 0.027$ \\
$23.34-23.40$ & $\ldots$ & $\ldots$ & $13.490 \pm 0.032$ \\
$24.34-24.40$ & $\ldots$ & $\ldots$ & $13.380 \pm 0.018$ \\
$25.37-25.40$ & $18.086 \pm 0.054$ & $15.290 \pm 0.048$ & $13.484 \pm 0.062$ \\
\hline
\end{tabular}

TABLE 3

Line Features in the Infrared Spectra

\begin{tabular}{lccc}
\hline \hline \multicolumn{1}{c}{ Id. $(\lambda)$} & observed $\lambda$ & E.W. $(\AA)$ & FWHM $(\AA)$ \\
\hline He I (2.0587) & 2.0558 & -8.83 & 37.3 \\
Br gamma (2.1661) & 2.1600 & -2.16 & 19.14 \\
He II $(2.1891)$ & 2.1895 & -4.65 & 39.25 \\
Na I $(2.20624-2.20897)$ & 2.2044 & -4.91 & 35.2 \\
\hline
\end{tabular}




\section{TABLE 4}

\section{Results of the Fit to the GIS-PCA-HEXTE SpeCtra}

\begin{tabular}{|c|c|c|c|c|c|}
\hline Parameter & State I & State II & State III & $\begin{array}{c}\text { State IV } \\
\text { (April 22) }\end{array}$ & $\begin{array}{c}\text { State IV } \\
\text { (April 23) }\end{array}$ \\
\hline$N_{\mathrm{H}}\left(10^{22} \mathrm{~cm}^{-2}\right)$ & $4.8 \pm 0.1$ & $4.6 \pm 0.1$ & $4.2 \pm 0.1$ & $4.0 \pm 0.1$ & $4.1 \pm 0.1$ \\
\hline$k T_{\text {in }}(\mathrm{keV})^{\mathrm{a}}$ & $1.46 \pm 0.05$ & $1.28 \pm 0.04$ & $0.85 \pm 0.02$ & $0.70 \pm 0.03$ & $0.84 \pm 0.05$ \\
\hline$R_{\text {in }}(\mathrm{km})^{\mathrm{a}}$ & $30 \pm 2$ & $32 \pm 2$ & $52 \pm 3$ & $61 \pm 7$ & $49 \pm 8$ \\
\hline$A^{\mathrm{b}}$ & $3.6 \pm 0.4$ & $2.8 \pm 0.2$ & $1.72 \pm 0.04$ & $2.16 \pm 0.04$ & $2.44 \pm 0.09$ \\
\hline$\Gamma^{\mathrm{b}}$ & $2.60 \pm 0.10$ & $2.44 \pm 0.06$ & $2.52 \pm 0.05$ & $2.41 \pm 0.05$ & $2.54 \pm 0.09$ \\
\hline$E_{\text {break }}(\mathrm{keV})^{\mathrm{b}}$ & $6.1 \pm 0.5$ & $6.3 \pm 0.2$ & $6.6 \pm 0.2$ & $6.5 \pm 0.2$ & $6.7 \pm 0.3$ \\
\hline$E_{\text {fold }}(\mathrm{keV})^{\mathrm{b}}$ & $115_{-32}^{+56}$ & $78_{-12}^{+17}$ & 1000 (fix) & $200_{-40}^{+70}$ & $290_{-120}^{+530}$ \\
\hline$\Omega / 2 \pi^{\mathrm{c}}$ & & & $0.88 \pm 0.10$ & $0.64 \pm 0.08$ & $0.66 \pm 0.14$ \\
\hline$\xi^{\mathrm{c}}$ & & & $<0.02$ & $<20$ & $<73$ \\
\hline E.W. $(6.5 \mathrm{keV})(\mathrm{eV})^{\mathrm{d}}$ & & & $<12$ & $<13$ & $<21$ \\
\hline$\tau_{\text {edge }}(9.3 \mathrm{keV})^{\mathrm{e}}$ & & & $0.08 \pm 0.03$ & $0.05 \pm 0.02$ & $0.04 \pm 0.03$ \\
\hline $2-10 \mathrm{keV}$ Flux $\left(10^{-8}\right)^{\mathrm{f}}$ & 2.23 & 1.54 & 0.77 & 0.80 & 0.97 \\
\hline $10-200 \mathrm{keV}$ Flux $\left(10^{-8}\right)^{\mathrm{f}}$ & 0.98 & 0.88 & 0.88 & 1.09 & 1.10 \\
\hline$\chi^{2} /$ dof & $174 / 154$ & $178 / 154$ & $163 / 151$ & $142 / 150$ & $103 / 150$ \\
\hline
\end{tabular}

a The component of the multi-color disk model (Mitsuda et al. 1984): $k T_{\text {in }}$ and $R_{\text {in }}$ is the (apparent) innermost temperature and radius, respectively. To calculate $R_{\text {in }}$ a distance of $11 \mathrm{kpc}$ and an inclination of $66^{\circ}$ are assumed.

b The component of a broken power law: $A$ is the normalization at $1 \mathrm{keV}$ in units of photons $\mathrm{cm}^{-2} \mathrm{~s}^{-1} \mathrm{keV}^{-1}, \Gamma$ is the photon index above the break energy $E_{\text {break }}$, below which the photon index is fixed at 1.65. High energy cutoff ("highecut" in XSPEC) is multiplied with an cutoff energy of $17 \mathrm{keV}$ (fixed) with a folding energy $E_{\text {fold }}$.

c The reflection component by Magdziarz \& Zdziarski (1995, "pexriv" in XSPEC). $\Omega$ is the solid angle of the reflector and $\xi$ is the ionization parameter. The disk temperature, elemental abundance, and inclination are fixed at $3 \times 10^{4} \mathrm{~K}, 1$ solar, and $66^{\circ}$, respectively. The input spectrum is approximated by a cutoff power law model with a photon index and a folding energy of 2.4 and $200 \mathrm{keV}$ (State III), 2.0 and $70 \mathrm{keV}$ (State IV, April 22), and 2.2 and $80 \mathrm{keV}$ (State IV, April 23).

$\mathrm{d}$ The equivalent width of an iron-K emission line fixed at $6.5 \mathrm{keV}$ with a $1 \sigma$ width of $10 \mathrm{eV}$.

e The optical depth of the $9.3 \mathrm{keV}$ edge from H-like iron ions. An absorption line from the same ions are included with the parameters as measured by Chandra HETGS (Lee et al. 2001).

${ }^{\mathrm{f}}$ The absolute flux is normalized to the GIS value.

Note.-Errors are $90 \%$ confidence limits for a single parameter. 
Figure 1

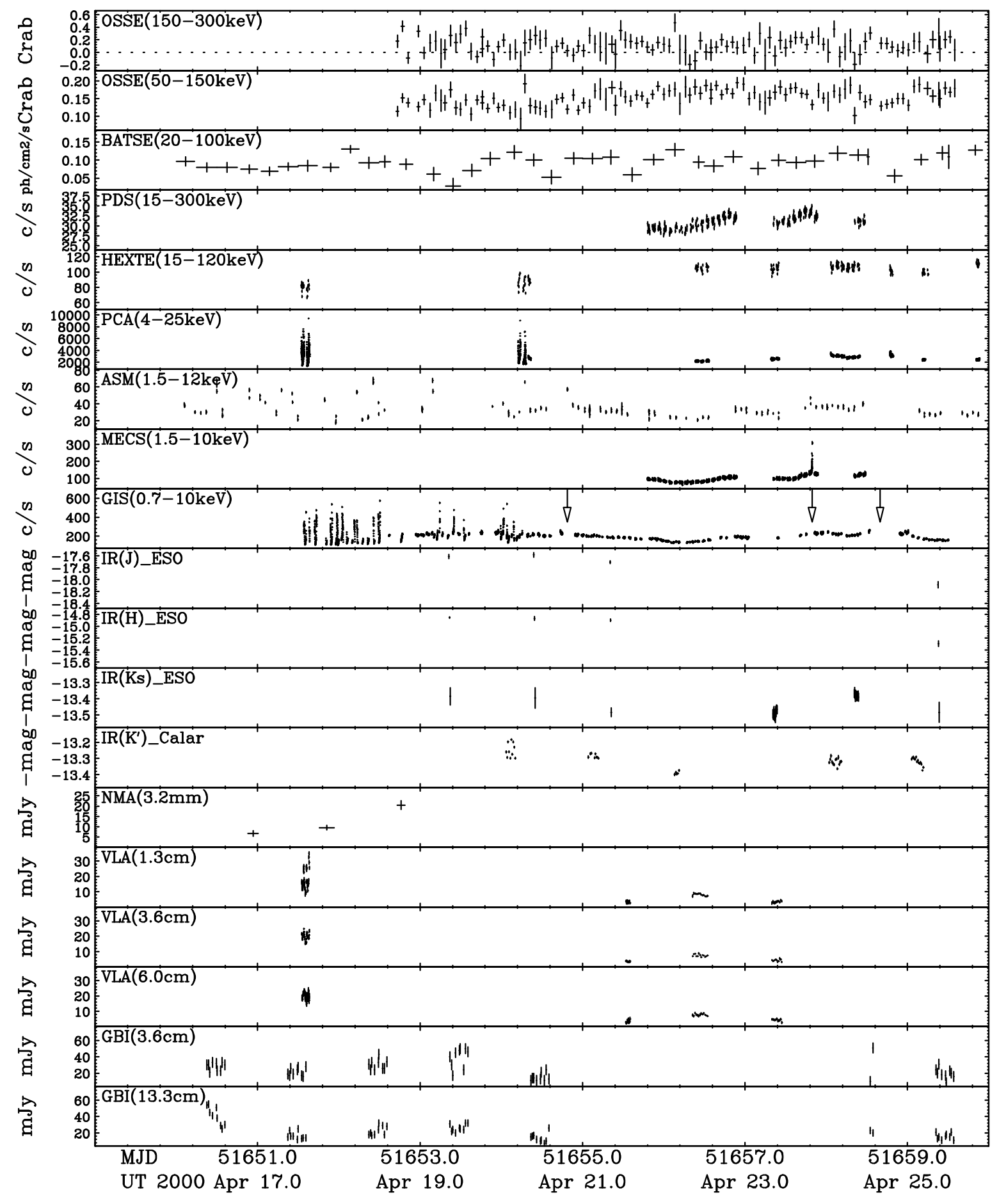


Figure 2

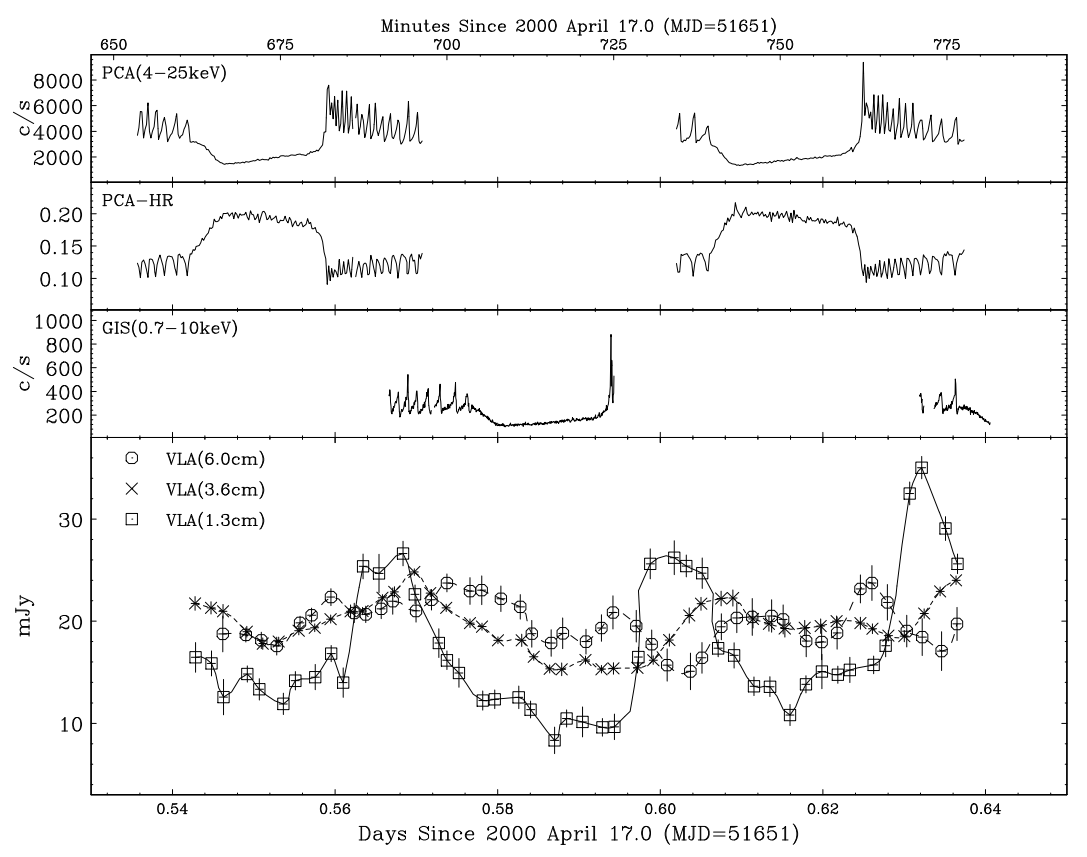

Figure 3

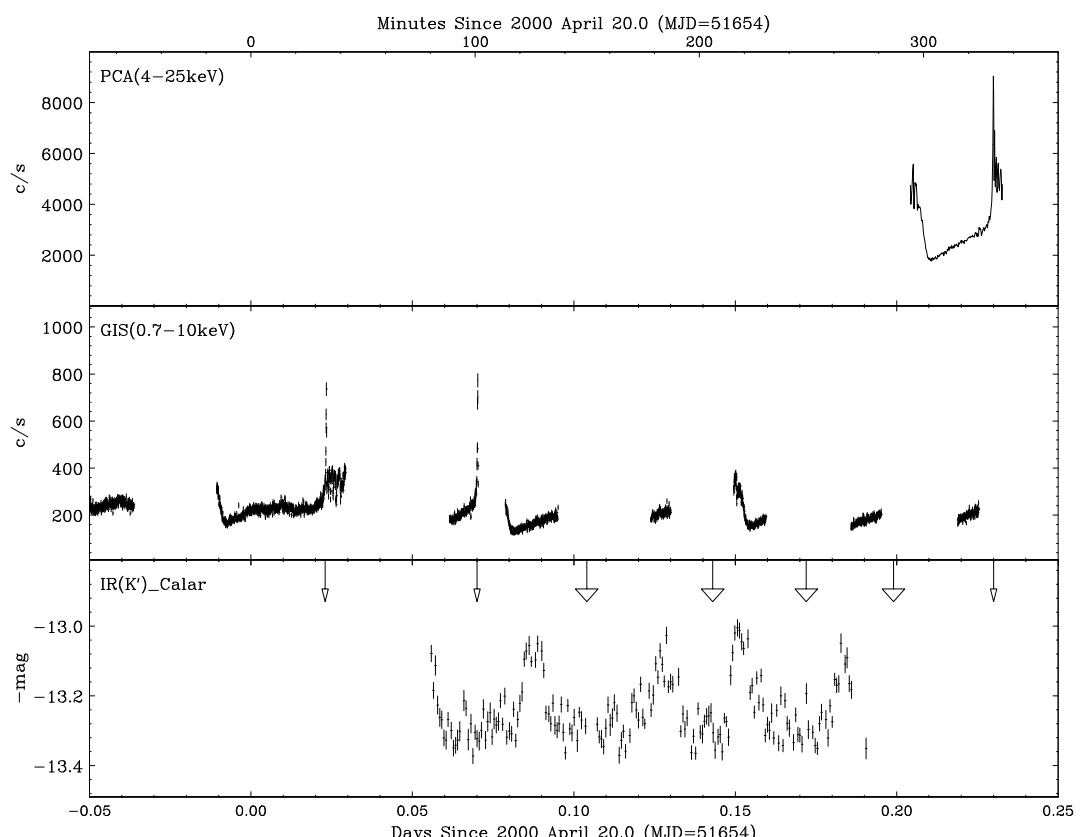


Figure 4

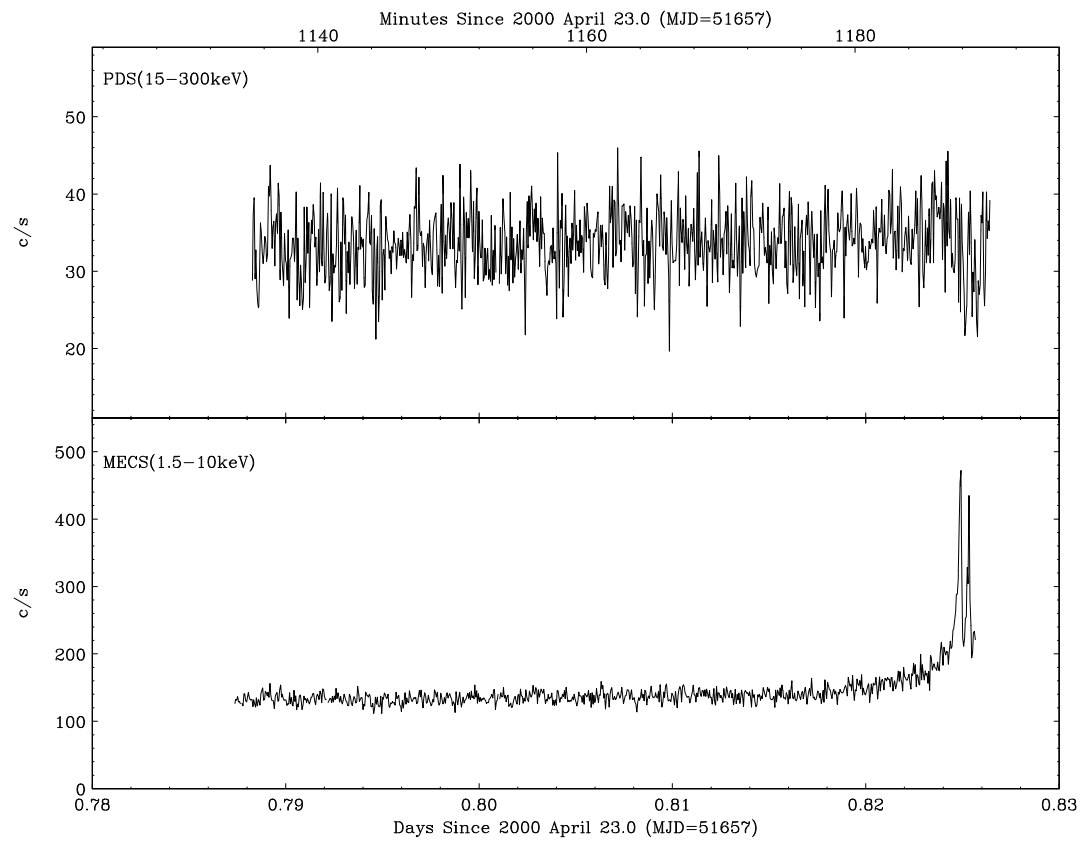

Figure 5

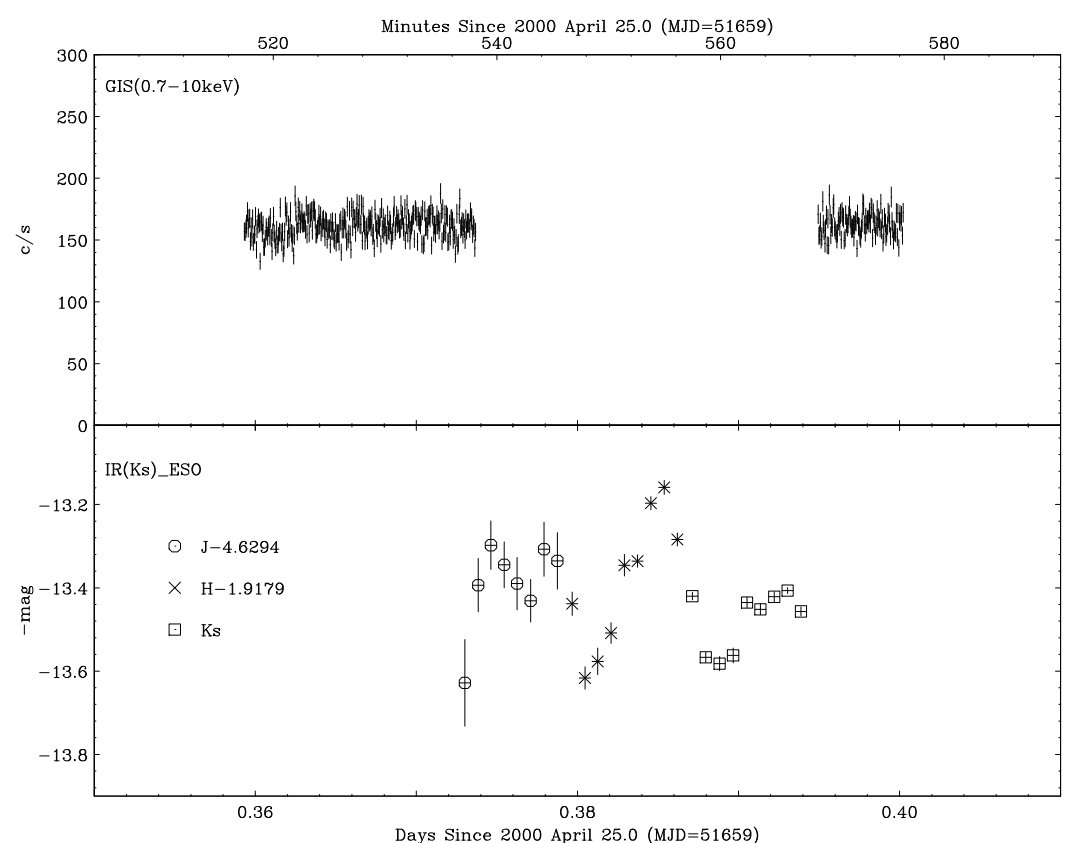


Figure 6

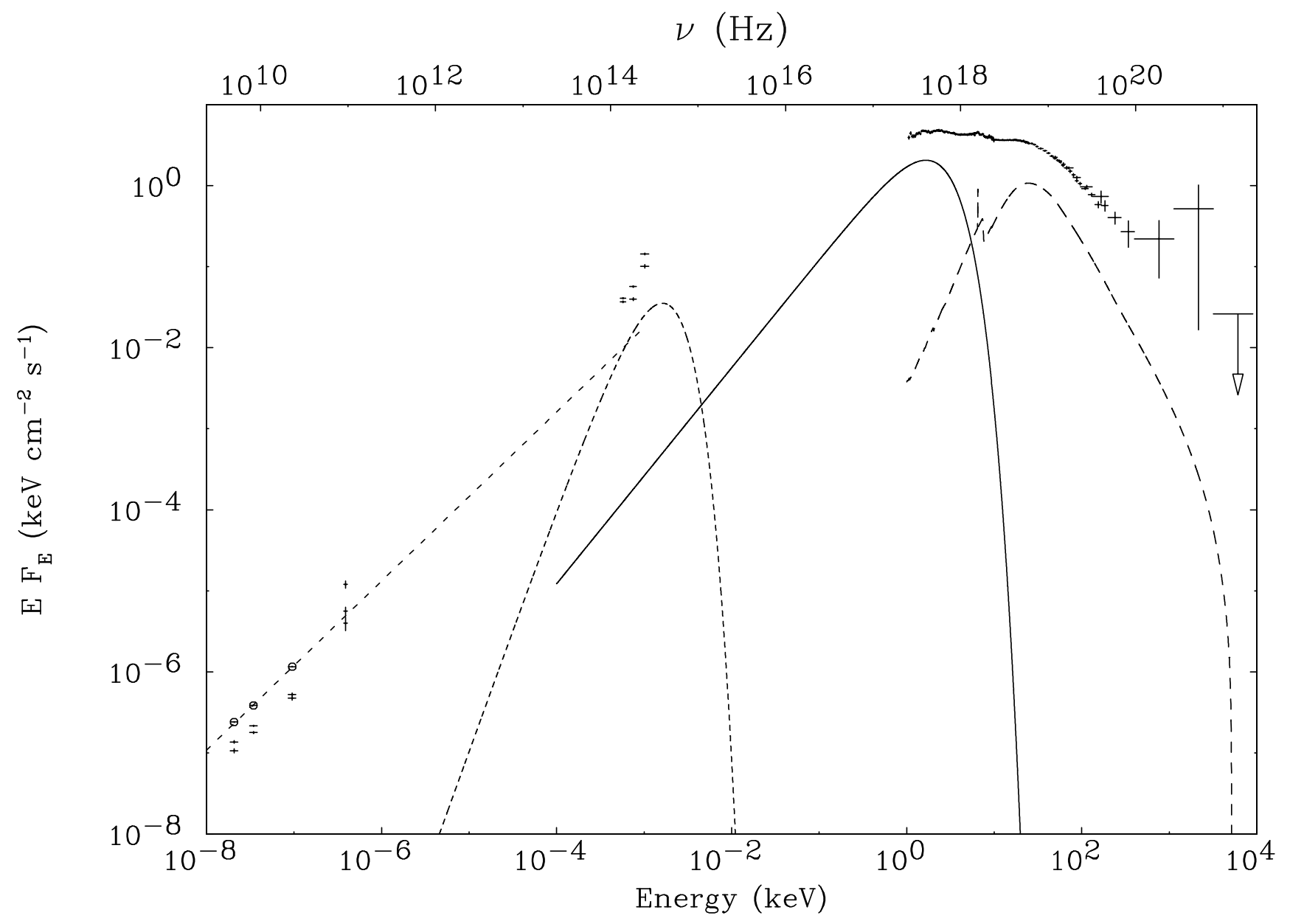


Figure 7
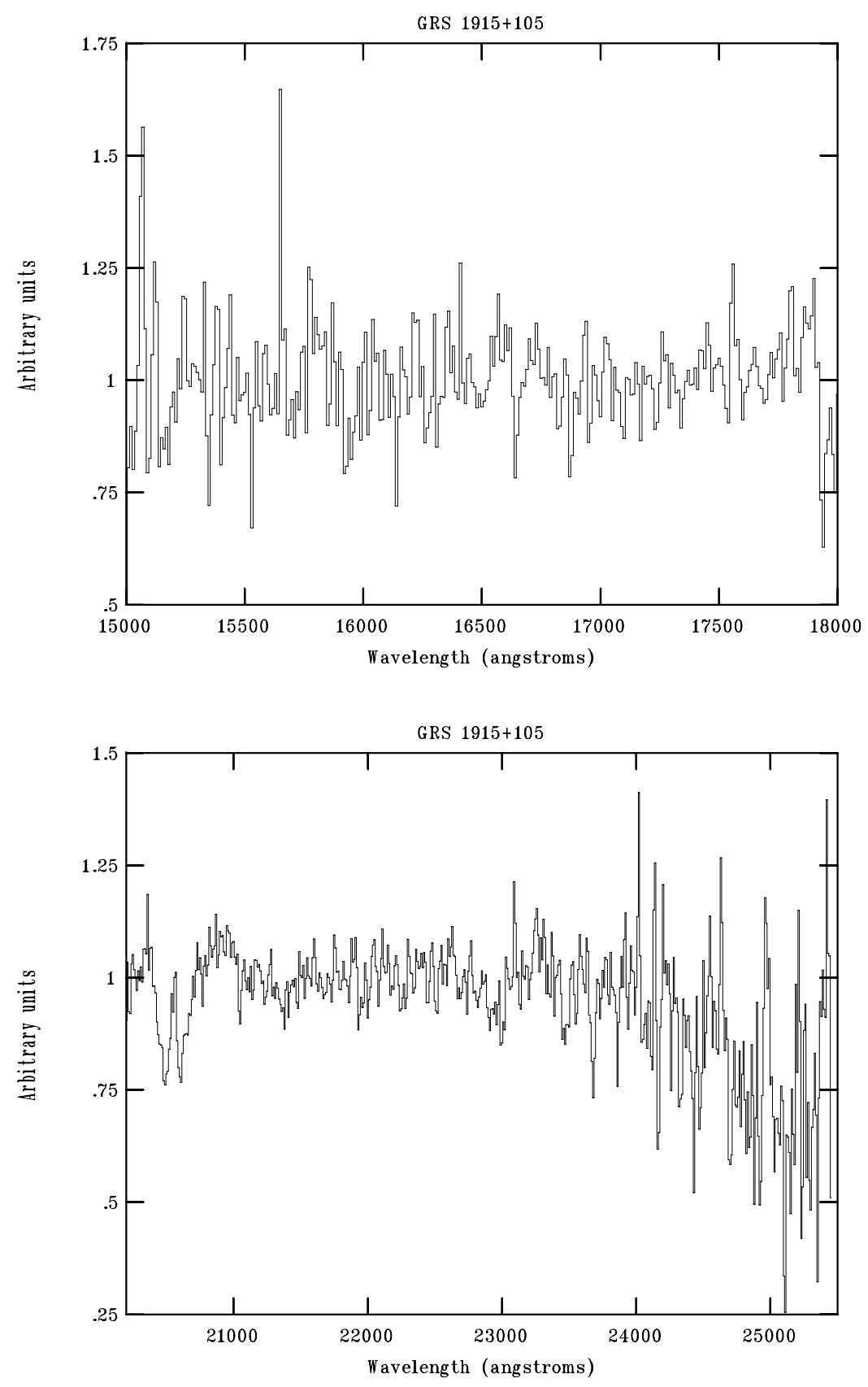
Figure 8

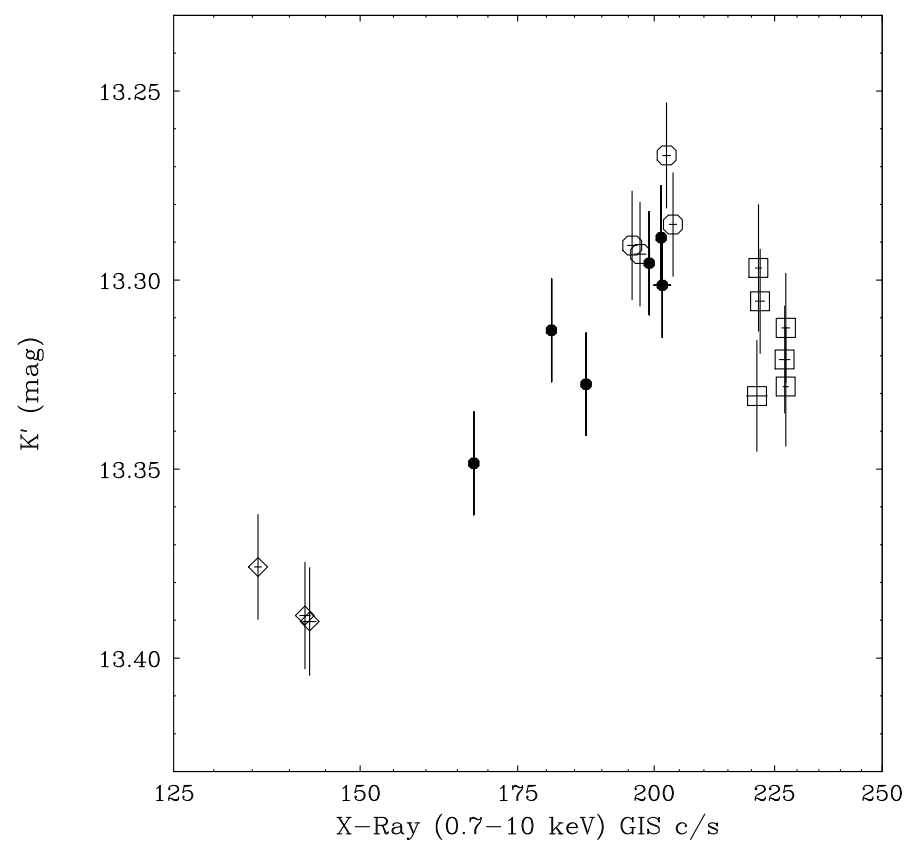

Figure 9

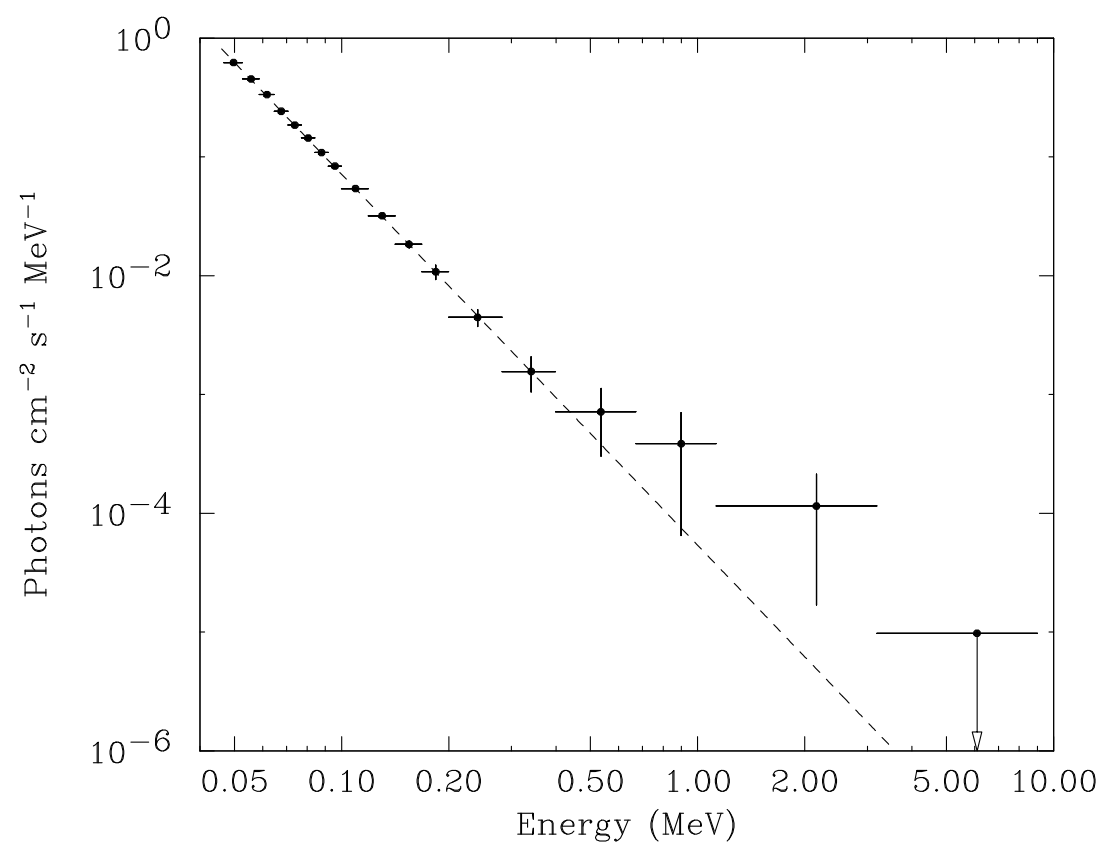


Figure 10

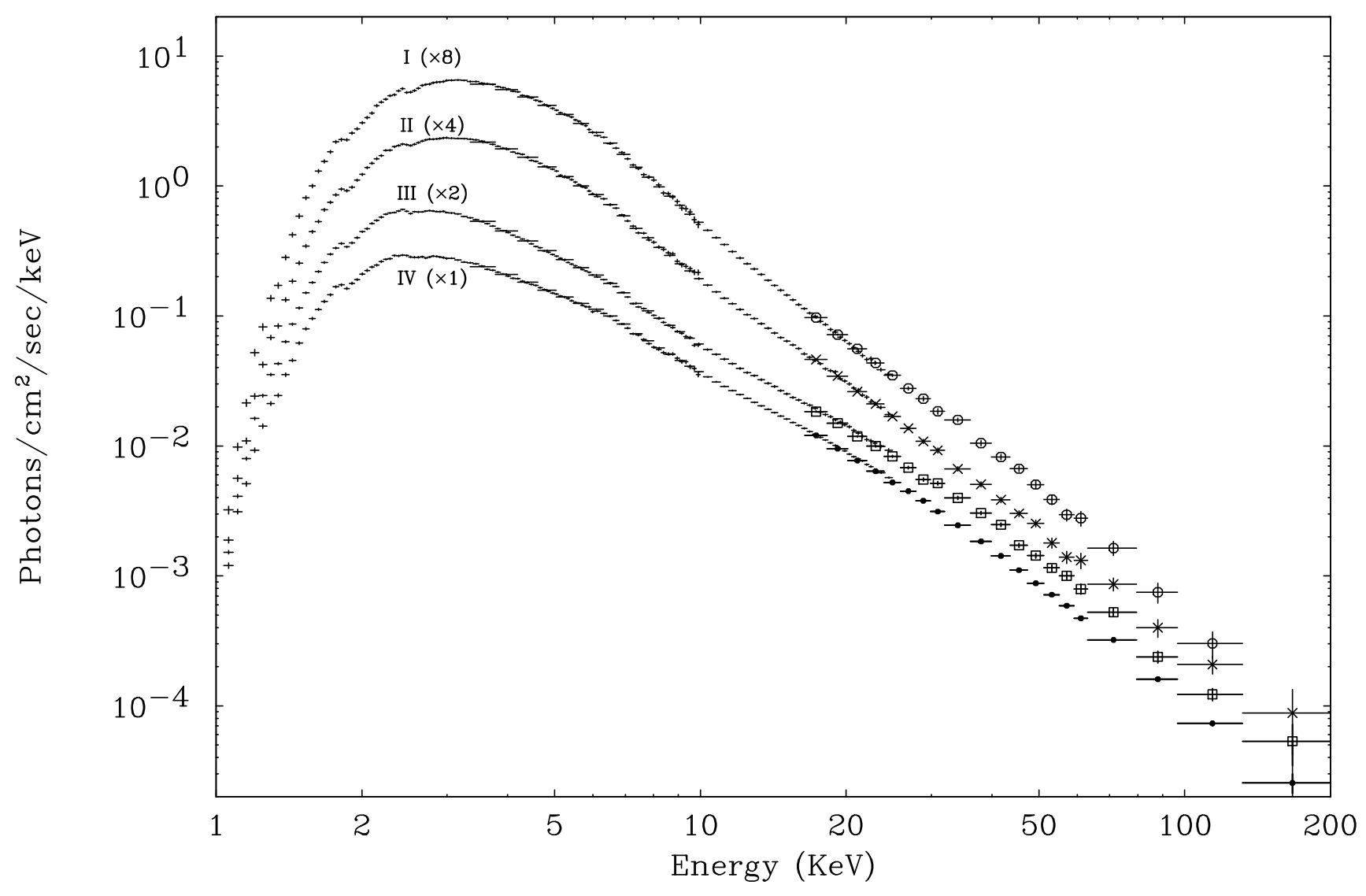

